\title{
Interfacial properties of selected binary mixtures containing n-alkanes
}

\author{
Erich A. Müller * Andrés Mejía *,1
}

Department of Chemical Engineering, Imperial College London, South Kensington Campus, London SW7 2AZ, United Kingdom

\begin{abstract}
We report the correlation and prediction of the subcritical vapour-liquid interfacial properties of three asymmetric binary mixtures composed of long n-alkanes in equilibria with a smaller solvent: hexane + decane, carbon dioxide + decane, and ethane + eicosane. The interfacial region is described by the complementary use of the Square Gradient Theory (SGT) and Molecular Dynamics (MD) simulations that allows the prediction of both the macroscopic and molecular level properties of the binary fluid mixtures. Calculations with SGT rely on the description of the vapour-liquid equilibria by means of the Statistical Associated Fluid Theory (SAFT) equation of state. MD simulations are performed in the canonical ensemble using united-atom potentials to probe coexisting phases and the accompanying interface simultaneously. In addition to the phase equilibrium compositions and interfacial tensions, other interfacial properties, such as concentration profiles along the interfacial region, surface activities, and relative Gibbs adsorption isotherms at the interfaces have been obtained from a combination of SGT and MD. The entropy and surface enthalpy change of surface formation are also calculated for the case of ethane + eicosane mixture, where we report three different isothermal conditions. While pure component data are used to fit model parameters, mixture results are predictions. When possible, results are compared to available experimental data and quantitative agreement is observed throughout. The particularly high excess adsorption in the interface of the smaller solvents, $\mathrm{CO}_{2}$ and ethane, is noted.
\end{abstract}

Keywords: Gradient Theory, Molecular Dynamics, Hydrocarbon Mixtures, SAFT, paraffins, EOR, C10, C20, Test Area Method.

\footnotetext{
* To whom correspondence should be addressed.e_mail: e.muller@imperial.ac.uk, amejia@udec.cl

${ }^{1}$ Permanent address: Departamento de Ingeniería Química, Universidad de Concepción, POB 160 - C, Correo 3, Concepción, Chile.
} 


\section{INTRODUCTION}

This work highlights the simultaneous application of a theoretical method and molecular simulations for the description of the interfacial behaviour of selected asymmetric hydrocarbon mixtures, where a low molecular weight solvent is in contact with a larger n-alkane (paraffin). These mixtures are interesting from a fundamental point of view, due to the effects of asymmetry on the interfacial behaviour, which in itself may trigger phase separation and in extreme cases, liquid crystal-like behaviour. Additionally these type of asymmetric mixtures currently enjoy renewed interest from the perspective of their importance in enhanced oil recovery and/or sequestration of $\mathrm{CO}_{2}$ in oil reservoirs [1]. In these scenarios, wettability between phases and interfacial phenomena play a key role, and the thermophysical information must be known in a broad range of temperature, pressure, and compositions [2-5].

Although one can rightfully argue that there can be no substitutes for well-performed experiments, these are notoriously expensive and no single existing methodology is self-sufficient to obtain a full description of the phase equilibrium and interfacial properties. Furthermore, there is a particular difficulty in obtaining experimental information at the molecular level. It is here that both theory and simulations can provide complementary information and physically meaningful extrapolation of experimental data. In some of our previous papers we have demonstrated that the combination of theory and experimental data provide a route to a rational experimental design, allowing the experimental results to be modelled and explained in terms of the theory [6-9]. Similarly, we have combined theory and simulation for the case of model mixtures $[10,11]$ where, by using the same intermolecular potentials in both, we were able to unambiguously understand and quantify the limitations of the theory. Finally, we also have applied molecular simulations to directly model the interfacial properties of reservoir fluid mixtures $[12,13]$. Following these lines, the main goal of this work is to combine, in a single study theory, simulations and available experimental data for the case of three prototypical hydrocarbon mixtures, namely hexane + decane, carbon dioxide + decane, and ethane + eicosane.

\section{Theory}


Several semi-empirical and theoretical approaches are available for estimating the interfacial tension (IFT), $\gamma$, of mixtures,. The parachor method [14], density functional theories [15, 16] and recently density functional perturbation theories [17]. From a theoretical viewpoint, one of the most elegant and powerful theories for fluid mixtures is the Square Gradient Theory for fluid interfaces (SGT). The SGT was originally developed by van der Waals in 1893 [18] and reformulated later by Cahn and Hilliard [19]. Briefly, SGT describes a continuous evolution of the Helmholtz energy density along the interfacial region, from which interfacial properties (i.e. concentration profiles, interfacial thickness, surface activity, Gibbs's adsorption and interfacial tension) can be calculated. In principle, the Helmholtz energy density can be described from any equation of state (EoS), with the consequence that a single EoS can be used to predict both phase equilibrium and interfacial properties. Previous works demonstrated that the combination of the SGT with cubic type EoS [6-8, 20-27] as well as molecular based EoS [9,28-33] models is promising for correlating and/or predicting interfacial properties in

some pure hydrocarbon and hydrocarbon mixtures in a wide range of temperatures, pressures and concentrations. Both the SGT and available EoS models require at some stage experimental data for pure component parameter fitting.

The Square Gradient Theory for fluid interfaces (SGT) has been described extensively in the literature (see Refs. 6-10; 20-33, additional description and numerical procedures related to SGT implementation can be found in Refs. 34-38). In this application we focus on strongly asymmetric mixtures, where custom-built physically based EoS should prove to have an advantage. The Statistical Association Fluid Theory (SAFT) is one example of such developments. The SAFT EoS has been broadly summarised in Refs. 39-44. SGT and SAFT are well known, and only the equations that are central to this work will be recalled in this section. In spite of this, it is noteworthy that only a few papers are devoted to use SGT approach with the SAFT EoS for the hydrocarbon mixtures [9, 30-33].

\subsection{The Square Gradient Theory for fluid interfaces}

According to the SGT formalism, the concentration of species along the interface region obeys the condition of minimum Helmholtz energy $(A)$. For the case of a planar interface between two bulk fluid phases in equilibrium $(\alpha, \beta)$ this constraint of minimum Helmholtz energy can be described, for a 
binary mixture, by the following set of ordinary differential equations (see Refs. 7 to 9, 20, 21 and 34 to 38$)$ :

$$
\begin{aligned}
& c_{11} \frac{d^{2} \rho_{1}}{d z^{2}}+c_{12} \frac{d^{2} \rho_{2}}{d z^{2}}=\mu_{1}-\mu_{1}^{0} \\
& c_{21} \frac{d^{2} \rho_{1}}{d z^{2}}+c_{22} \frac{d^{2} \rho_{2}}{d z^{2}}=\mu_{2}-\mu_{2}^{0}
\end{aligned}
$$

The boundary conditions of Eq. 1 are given by the limits of the bulk fluid phase equilibrium

$$
\rho_{\mathrm{i}}(\mathrm{z} \rightarrow-\infty)=\rho_{\mathrm{i}}^{\alpha} \quad \text { and } \quad \rho_{\mathrm{i}}(\mathrm{z} \rightarrow+\infty)=\rho_{\mathrm{i}}^{\beta}
$$

In Eq. 1 and 2, $\rho_{i}$ is the molar concentration of species $i$. It is related with the molar concentration of the mixture $(\rho)$ by the molar fraction $\left(x_{i}\right): \rho_{i}=x_{i} \rho . \rho_{i}{ }^{\alpha}, \rho_{i}{ }^{\beta}$ corresponds to the molar concentration of component $i$ in the $\alpha$ and $\beta$ bulk phases, respectively. $c_{i j}$ is the cross influence parameter $\left(c_{i j}=c_{j i}\right), z$ is a coordinate normal to the interface, and $\mu_{i}$ is the chemical potential of species $i$. The superscript 0 in $\mu_{i}$ denotes that it is evaluated at the phase equilibrium condition of the bulk phases $(\alpha, \beta)$. $\mu_{i}$ can be related with $\rho_{i}$ through the use of an equation of state (EoS). Integration of Eq. 1 allows quantification of the population of species at the interface $\left(\rho_{i}(z)\right)$ from which the surface activity (or absolute adsorption / desorption of species along the interface region), relative Gibbs adsorption and the interfacial tension can be calculated.

The accumulation of a species $i$ at the interface region is characterized by the condition, $d \rho_{i} / d z=0$, and it may be positive or negative. The positive surface activity reflects absolute adsorption of species along the interface region and is reflected in a negative second derivative, $d^{2} \rho_{i} / d z^{2}<0$. Conversely, the negative surface activity denotes desorption of species along the interface region and its condition is given by $d^{2} \rho_{i} / d z^{2}>0$. The relative Gibbs adsorption isotherm of a species $i$ relative to a species $j\left(\Gamma_{i j}\right)$ can be expressed in terms to $\rho_{i}(z)$ by the following integral equation [45]:

$\Gamma_{i j}=\int_{-\infty}^{z_{0}^{j}}\left[\rho_{i}(z)-\rho_{i}^{\alpha}\right] d z+\int_{z_{0}^{j}}^{+\infty}\left[\rho_{i}(z)-\rho_{i}^{\beta}\right] d z$ 
In Eq. 3, $z_{0}{ }^{\mathrm{j}}$ is the localization of the divide position relative to a species $j . z_{0}{ }^{\mathrm{j}}$ is calculated from Eq. 3 considering that species $j$ does not have adsorption along the interfacial region. In other words, Eq. 3 is solved for the case that $\Gamma_{j j}=0$.

According to Rowlinson and Widom [45], $\Gamma_{i j}$ can be evaluated, alternatively, as

$\Gamma_{i j}=-\left(\frac{\partial \gamma}{\partial x_{i}}\right)_{T, P}\left(\frac{\partial \mu_{i}^{0}}{\partial x_{i}}\right)_{T, P}^{-1}$

In the latter approach, $\gamma$ is the interfacial tension between $\alpha$ and $\beta$ bulk phases. Comparing Eq. 3 to Eq. 4 , it is possible to observe that in Eq. 3 we use the concentration profile along the interfacial region with an explicit definition of the divide position and in Eq. 4 we use the isothermal variation of $\gamma$ and $\mu_{i}^{0}$ with $x_{i}$ with an implicit and theoretical definition of the divide position. Both approaches (Eq. 3 and 4) can be shown to give rise to qualitatively different values of $\Gamma_{i j}$ due to the ill-definition of the divide position. The reader is refered to Refs. $[45,46]$ for a complete discussion on this topic.

There is no preferred approach to calculate $\Gamma_{i j}$, however, the application of Eq. 3 is straightforward in MD simulations, and Eq. 4 is the usual route used to process experimental measurements, especially where the chemical potential can be modelled in terms to activity coefficients. (see Ref. 47 as an example).

In the context of SGT, $\gamma$ can be calculated from the following integral expression:

$\gamma=\int_{-\infty}^{\infty}\left[c_{11}\left(\frac{d \rho_{1}}{d z}\right)^{2}+2 c_{12} \frac{d \rho_{1}}{d z} \frac{d \rho_{2}}{d z}+c_{22}\left(\frac{d \rho_{2}}{d z}\right)^{2}\right] d z$

Inspection of Eqs. 1 and 5 reveals that the calculation of $\rho_{i}(z), \Gamma_{i j}$ and $\gamma$ depend on the EoS model and on the $c_{i j}$ values. The role of a specific EoS is to provide analytical relations for chemical potential and to predict the equilibrium state at which phases coexist. As we will describe in the next section, $\mu_{i}$ is calculated, in this work, from a version of the SAFT - EoS.

In this work, $c_{i j}$ is calculated using the procedure suggested by Carey et al.[20,34] Briefly, for the case of pure fluids, $c_{i i}(i=j)$ is calculated at the boiling temperature from experimental $\gamma$ data using Eq. 5 for 
the case of pure fluids. The temperature dependence of $c_{i i}$ is usually correlated using the following linear function,

$$
c_{i i}=c_{i i}^{0}+c_{i i}^{l} T
$$

For the case of mixtures $(i \neq j), c_{i j}$ is obtained by averaging the pure component influence parameters according to

$$
c_{i j}=\left(1-\chi_{i j}\right) \sqrt{c_{i i} c_{j j}}
$$

where $\chi_{i j}$ is a symmetric adjustable parameter that, in turn, may be obtained from the fit of experimental $\gamma$ data of mixtures. It is important to point out that for some binary mixtures a value of $\chi_{i j}$ $=0$ is adequate to model their interfacial tensions. In this work, $\chi_{i j}$ is set to zero, rendering the procedure predictive in nature. In this case, Eqs. 1 simplifies to:

$$
\sqrt{c_{11}}\left[\mu_{2}-\mu_{2}^{0}\right]=\sqrt{c_{22}}\left[\mu_{1}-\mu_{i}^{0}\right]
$$

Specific details that relate to the numerical procedure for calculating $\rho_{i}(\mathrm{z})$, surface activity, and $\gamma$ from Eq. 1 (or Eq. 8) and Eq. 5, respectively, have been described in detail previously [34-37].

It is possible to define, the entropy $\left(\Delta s^{\gamma}\right)$ and surface enthalpy $\left(\Delta h^{\gamma}\right)$ change of surface formation from the following derivative expressions $[45,46,48]$ :

$$
\begin{aligned}
& \Delta s^{\gamma}=-(\partial \gamma / \partial T)_{P, x} \\
& \Delta h^{\gamma}=\gamma+T \Delta s^{\gamma}
\end{aligned}
$$

\subsection{The Statistical Associated Fluid Theory (SAFT) Model}

The Statistical Associated Fluid Theory (SAFT) is a generalized model that allows the development of physically based EoS for chain-like fluids where association, of the type found in hydrogen bonding or 
charge transfer processes, is present. Several implementations of the theory have lead to different versions of the final equations. The improvement obtained by switching from one version to the other is generally marginal. However, the parameters of SAFT-like equations are more molecular-based and better behaved than the cubic EoS that they tend to replace. The reader is referred to reviews on the topic for further discussions $[40-42,44]$.

Here we employ the SAFT model as parametrized by Huang and Radosz [49]. As with all SAFT models, it is based on a perturbative approach with additive contributions to the Helmholtz energy density $(a=A / V)$, which for a non-associating chain fluid becomes:

$a(\rho, T)=a^{R}+a^{i g}=\left(a^{h s}+a^{\text {chain }}+a^{\text {disp }}\right)+a^{i g}$

where $a^{R}$ is the residual Helmholtz energy density, and $\mathrm{a}^{i g}$ is the ideal gas reference. $\mathrm{a}^{R}$ is formed of three additive contributions to the Helmholtz energy density: $a^{h s}$ represents repulsion interactions between molecules, $a^{\text {chain }}$ accounts for the formation of molecule chains, and $a^{\text {disp }}$ represents dispersion attraction between molecules. Pure components are characterized by three parameters: the molecular chain length (or number of segments) $m_{i}$, the segment volume $v_{i}^{o o}$, the temperature dependent well depth energy $u_{i}^{o}$. A fourth constant $e / k_{B}$, related to the Pitzer's acentric factor and the critical temperature, was set 40 for carbon dioxide and 10 for the other molecules. The SAFT-EoS is extended to mixtures by applying quadratic mixing rules with an interaction parameter $\left(k_{i j}\right)$ to the dispersion term. (see Ref. [49] for details)

From Eq. 11 and using the chemical potential definition [50], it is possible to obtain the following expression for the chemical potential of species $i$ in mixture:

$\mu_{i}=\left(\frac{\partial a}{\partial \rho_{i}}\right)_{T, V, \rho_{j \neq i}}=\left(\frac{\partial a^{R}}{\partial \rho_{i}}\right)_{T, V, \rho_{j}}+R T \ln \left(\rho_{i} \Lambda_{i}^{3}\right)$

where $\Lambda_{i}$ is the Broglie's wavelength. In this work, Eq. 12 will be used to calculate the phase equilibrium between the bulk phases as well as interfacial properties (see Eqs. 1 and 8).

\section{Simulations}


The most common method for studying interfaces via molecular simulation is to include in a simulation cell both intervening bulk phases and the corresponding interface. Such an inhomogeneous system is not always straightforward to set up and analyze, as a sensible system size is needed to provide bulk phases and an interface (in fact, due to the periodic boundary conditions commonly employed, most setups actually present two interfacial regions) with a significant amount of molecules. Until recently, these simulations were confined to the realm of large-scale hardware. This is not the case anymore [51]. For the most cases, systems may be set up in the canonical ensemble, where the number of molecules, $N$, the temperature $T$ and the total system volume $(V)$ are kept constant. If the system is placed at appropriate thermodynamic conditions, the two phases and the interface may be simultaneously monitored. Equilibrium is guaranteed by performing either Monte Carlo steps or by letting the system evolve through molecular dynamics. Molecular Dynamics (MD) is arguably the method of choice for asymmetric mixtures, as the simulation method is applicable to both dilute and dense phases, easily parallelized, and suited for simulating very large complex molecules. Simulations may be speeded up if the initial system is held at a high temperature, where a unique homogeneous well-mixed phase is present. Quenching to the desired temperature can be done instantaneously and the system is then allowed to evolve under NVT conditions until equilibration is reached through diffusive mass transport [51].

Special mention is made here of the GCMC method, in which the system is envisioned to be in equilibrum with a reservoir of molecules at the same chemical potential as the system in question. By means of particle interchanges between the system and the reservoir, diffusional equilibrium is reached. Internal equilibrium is reached by additional MC moves on the particles inside the system. GCMC can thus be used effectively to construct the same type of heterogeneous bulk plus interfacial system as descried in the previous paragraph. It brings the added benefit that the interfacial tension can be calculated directly from the density histograms [52, 53]. However, MC methods are notoriously cumbersome for larger molecules and require special techniques (such as Configurational-Bias Monte Carlo [54-56]) to deal with dense or elongated molecules.

\subsection{Intermolecular potential Models}

The hydrocarbons considered here (ethane, hexane, decane, and eicosane) are modelled using unitedatom NERD [57] model. In this scheme, bond stretching and bond bending interactions are modelled in terms of harmonic potential, 
$U\left(r_{i j}\right)=\frac{k_{r}}{2}\left(r_{i j}-r_{0}\right)^{2}$

$U(\theta)=\frac{k_{\theta}}{2}\left(\theta-\theta_{0}\right)^{2}$

where $r_{i j}$ is the bond distance between two united-atoms, and $\theta$ is the bond angle between three consecutive united-atoms. The subscript 0 denotes their equilibrium value, and $k_{r}$ and $k_{\theta}$ are the corresponding spring constant. The constant values in Eq. 13 are $k_{r} / k_{B}=96500 \mathrm{~K} / \AA^{2}, r_{0}=1.54 \AA$, where $k_{B}$ is Boltzmann's constant. For the case of hexane, decane, and eicosane molecules, the constant values in Eq. 14 are $k_{\theta} k_{B}=62500 \mathrm{~K} / \mathrm{rad}^{2}, \theta_{0}=114.0^{\circ}$. For the long chain hydrocarbons, the torsional potential is represented by [58]

$U(\phi)=c_{1}(1+\cos \phi)+c_{2}(1-\cos 2 \phi)+c_{3}(1+\cos 3 \phi)$

where $\phi$ is the dihedral angle between four consecutive united-atoms, $c_{1}, c_{2}$, and $c_{3}$ have the following values: $\mathrm{c}_{1} / k_{B}=355.03 \mathrm{~K}, \mathrm{c}_{2} / k_{B}=-68.18 \mathrm{~K}$, and $\mathrm{c}_{3} / k_{B}=791.32 \mathrm{~K}$.

In this work the interaction between two united-atoms in different molecules or separated by more than three bonds within a molecule is given by a Lennard - Jones potential

$U\left(r_{i j}\right)=4 \varepsilon_{i j}\left[\left(\sigma_{i j} / r_{i j}\right)^{12}-\left(\sigma_{i j} / r_{i j}\right)^{6}\right]$

where $r_{i j}$ is the distance between united-atoms $i$ and $j . \varepsilon_{i j}$ is the energy parameter of the interaction, whereas $\sigma_{i j}$ is the Lennard - Jones size parameter. Unlike interactions are calculated by using the Lorentz - Berthelot mixing rules:

$\varepsilon_{i j}=\sqrt{\varepsilon_{i i} \varepsilon_{j j}} \quad, \quad \sigma_{i j}=\left(\sigma_{i i}+\sigma_{j j}\right) / 2$

In this work, the Lennard - Jones parameters have the following values: ethane: $\varepsilon_{C H 3} / k_{B}=112.20 \mathrm{~K}$, $\sigma_{C H 3}=3.64 \AA$. For the other n- alkanes (hexane, decane, and eicosane): $\varepsilon_{C H 3} / k_{B}=104.0 \mathrm{~K}, \varepsilon_{C H 2} / k_{B}=$ $45.8 \mathrm{~K}, \sigma_{C H 3}=3.91 \AA, \sigma_{C H 2}=3.93 \AA$. 
Carbon dioxide is modelled using a rigid model (EPM2 [59]). In this model, the length between atoms is fixed at $1.149 \AA$ and the interaction between two united-atoms in different molecules or separated by more than three bonds within a molecule is given by a combination of Lennard - Jones potential and Coulomb potential:

$$
U\left(r_{i j}\right)=4 \varepsilon_{i j}\left[\left(\frac{\sigma_{i j}}{r_{i j}}\right)^{12}-\left(\frac{\sigma_{i j}}{r_{i j}}\right)^{6}\right]+\frac{q_{i} q_{j}}{r_{i j}}
$$

$q_{i}$ and $q_{j}$ are the charges assigned to united-atom $i$ and $j$, respectively. The values used in Eq. 18 are given by: $\varepsilon_{C} / k_{B}=28.129 \mathrm{~K}, \varepsilon_{O} / k_{B}=80.507 \mathrm{~K}, \sigma_{C}=2.757 \AA, \sigma_{O}=3.033 \AA$, and $q_{C}=0.6512 \mathrm{e}, q_{O}=$ 0.3256 e. Unlike interactions are calculated by Eqs. 17.

\subsection{Simulation Details}

Molecular Dynamics simulations are performed on binary mixtures containing more than 4000 united atoms at conditions where the liquid vapour interface is present. The distribution of molecules $\left(N_{l}\right.$ and $N_{2}$ ) was set according to the mole fraction desired and the systems were set up in a way that the volume fractions of the bulk phases were comparable.

In this work, all simulations are started from a high temperature homogeneous one phase system that was quenched instantaneously to the simulation temperature. The simulation cell is a $L_{x} \times L_{y} \times L_{z}$ parallelepiped with periodic boundary conditions in all three directions. Table 1 summarizes the box dimensions used for each mixture. These values are chosen in order to have a cell large enough to accommodate the liquid and gas regions with enough molecules to ensure a sensible bulk phase and the corresponding two interfacial regions. $L_{z}$ was in general much larger than $L_{x}$ and $L_{y}$, and the interface spontaneously appears in the $x-y$ plane. In Table 1 , we also summarize the cut-off radius used for each mixture. These values are chosen in order to reduce the truncation and system size effects involved in the phase equilibrium and interfacial tension calculations (see Refs. [60-64] for a complete discussion of truncation effects).

In this work all simulations for phase equilibria and interfacial properties are performed by using the DL_POLY package [65]. Simulations are carried out using the Verlet leapfrog algorithm with a time step of 0.003 ps and the canonical ensemble is sampled by an NVE simulation. In addition, for the case of ethane + eicosane mixture, the NVT ensemble with Nosé - Hoover thermostat is used with a 
relaxation constant of 1.0 ps. Phase equilibrium conditions are compared to those obtained by Gibbs ensemble simulations performed with the GIBBS program [66]. No significant difference was apparent between the results from these three approaches. For the case of mixtures with electrostatic contributions, the Ewald technique with a convergence parameter of $0.15515 \AA^{-1}$ and a maximum value for the reciprocal lattice equal to 31 is used. After the initial temperature quenching, the systems are equilibrated for $2 \mathrm{~ns}$. After this equilibration stage, a production run at least another $2 \mathrm{~ns}$ is performed. The corresponding statistics are accumulated every 500 time steps.

Concentration profiles are calculated by dividing the system in 250 slabs along the $z$ direction. The molecular density profiles, $\rho_{i}(z)$, are obtained by assigning the position of each united atom center, $z_{i}$, to the corresponding slab and constructing the molecular density from mass balance considerations. Additionally, these profiles are displaced in order that the centre of mass of the system lies at the centre of the simulation cell. This displacement may avoid a possible smearing of the profiles due to fluctuations of the centre of mass. The equilibrium pressure is obtained using the normal component of the pressure tensor calculated via the virial route.

\subsection{Interfacial tension via the TA method}

For a given system that exhibits two bulk equilibrium phases separated by interfaces, interfacial tension has traditionally been calculated from a mechanical route, i.e. by calculating the components of the pressure tensor via the virial route and relating the difference of the component of the normal contribution to that of the component in the plane of the interface to the interfacial tension [67]. Recently, however, a perturbative method has been proposed, which circumvents some of the ambiguities of the aforementioned mechanical route. This method, referred to as the test area (TA) method (see Gloor et al. [68] Errington and Kofke [69] and Ghoufi et al. [70] for a review of this approach) is used herein.

In the TA method, the interfacial tension, $\gamma$, can be obtained from the change in the Helmholtz energy $(A)$ in the limit of an infinitesimal perturbation in the interfacial area $(\mathcal{A})$ in the constant NVT ensemble. Mathematically, $\gamma$ is given by

$$
\gamma=\left(\frac{\partial A}{\partial \mathcal{A}}\right)_{N V T}=\lim _{\Delta \mathcal{A} \rightarrow 0}\left(\frac{\Delta A}{\Delta \mathcal{A}}\right)_{N V T}=-\frac{k_{B} T}{\Delta \mathcal{A}} \ln \left\langle\exp \left(-\Delta U / k_{B} T\right)\right\rangle_{0}
$$


where $\Delta A$ is the infinitesimal perturbation in $A . \Delta U$ denotes the change in the configurational energy due to $\Delta A .\langle\ldots\rangle_{0}$ indicates an ensemble average carried out over an equilibrated state. In order to evaluate Eq. 19, the equilibrated system (state 0$)$ with interfacial area $\mathcal{A}_{0}\left(\mathcal{A}_{0}=2 L_{x, 0} L_{y, 0}\right)$ is perturbed by an infinitesimal change in the interfacial area. This perturbation translates the system to a new state (perturbed state or state 1) that has the same volume as the original state, but a different interfacial area. The new interfacial area in state 1 is $\mathcal{A}_{l}$, which is obtained using the following transformations $L_{x, l}=$ $L_{x, 0} \sqrt{ }(1+\xi)$, and $L_{y, l}=L_{y, 0} \sqrt{ }(1+\xi)$, where $\xi \ll 1$. With this transformation $\mathcal{A}_{l}=\mathcal{A}_{0}+\Delta \mathcal{A}$ and $\Delta \mathcal{A}=$ $L_{x, 0} L_{y, 0} \xi$. It is important to consider that doing the latter perturbation, $L_{z}$ needs to change from $L_{z, 0}$ to $L_{z, l}=L_{z, 0} /(1+\xi)$ in order to guarantee a constant volume condition. At each state $(0$ and 1$)$, the configurational energy of the system is calculated, and the difference $\Delta U=U_{I}-U_{0}$ is evaluated. In this work, the calculation of $\gamma$ is carried out by both expanding $(+\Delta \mathcal{A})$ and compressing $(-\Delta \mathcal{A})$ the interfacial area. The final value of $\gamma$ for a given $|\Delta \mathcal{A}|$ is the result of the average over these two perturbations. A plot of the corresponding Boltzmann average $\left\langle\exp \left(-\Delta \mathrm{U} / \mathrm{k}_{\mathrm{B}} \mathrm{T}\right)\right\rangle_{0}$ as a function of the perturbation $|\Delta \mathcal{A}|$ allows for the calculation of the limiting value at $\Delta \mathcal{A} \rightarrow 0$. In order to define the magnitude of the perturbation, we evaluate expansion and compression paths for $\xi$ ranging from $10^{-7}$ to $10^{-2}$. According to our results, we found that $\gamma$ is constant in the range $10^{-5}<\xi<10^{-3}$. The value of $\xi$ used in this work is $5 \times 10^{-4}$, which is commensurate with previous other similar application of the TA method $[68,71-75]^{\cdot}$ All the TA calculations are performed by post-processing an uninterrupted MD run, as the perturbations have no effect on the dynamics of the system.

\section{Results and Discussions}

The main objective of this work is to simultaneously predict the phase equilibria and interfacial properties for hydrocarbon mixtures from SGT and MD, and to compare their results, when it is possible, with experimental data. In order to obtain a correct characterization of phase equilibria and interfacial properties of these mixtures and to be able to directly compare these two approaches, we first defined a set of pure fluid parameters to appropriately represent the experimental data. In Table 2 we summarize the pure fluid parameters used in the SAFT model and SGT. The pure fluid parameters of the SAFT - EoS have been taken from Huang and Radosz [49], and the pure influence parameter of SGT $\left(c_{i i}\right)$ are fitted from experimental $\gamma$ data, which are taken from Landolt-Börnstein data base [76]. In this work we use a constant value of $c_{i i}$ for each fluid as they show weak temperature dependence. 
Mixture calculations are then effectively predictions from the theoretical models. For MD simulations, the potential parameters have been optimized to represent liquid properties (densities, vapour - liquid equilibria) of the pure fluids and again have been used here directly. The mixture results are a product of the transferability of the potentials.

\subsection{Hexane + Decane mixture}

The hexane (1) + decane (2) mixture is considered here as a base case to analyze the phase and interface behaviour in hydrocarbon mixtures. The vapour - liquid equilibria (VLE) and IFT for this mixture have been measured as well as predicted by means of the SGT. However, a complete description of its interfacial properties (i.e. concentration profiles along the interfacial region, surface activity, relative Gibbs adsorption isotherm) is still missing. Figure 1 shows VLE for this system at $308.15 \mathrm{~K}$ and $313.15 \mathrm{~K}$ along with experimental results reported by Marsh et al. [77] at $308.15 \mathrm{~K}$, SAFT calculations at $308.15 \mathrm{~K}$ and $313.15 \mathrm{~K}$ and MD results at $313.15 \mathrm{~K}$. Inspection of this Figure reveals that this zeotropic mixture exhibits a moderate positive deviations from ideal behaviour.

For the SAFT calculations, $k_{i j}$ is optimized using the available experimental information. $k_{i j}$ value as well as deviation statistics for VLE predictions are summarized in Table 3. As expected, the SAFT model is reliable for correlating and predicting the VLE in the whole mole fraction range with a low absolute average deviation in vapour pressure and vapour mole fraction. Table 4 summarizes the MD results for this mixture. The MD results show a fairly good agreement to SAFT predictions with an absolute average deviation (AAD) in vapour pressure of $4.28 \%$, and $\Delta y=0.45 \%$. In addition to $P-x$ - $y$ diagram shown in Figure 1, Figure 2 shows the VLE in a density - pressure diagram at $313.15 \mathrm{~K}$. From this Figure, we can observe that MD and the SAFT calculations show the same trends in the bulk phases.

Figure 3 shows the interfacial tension of this mixture as a function of the liquid mole fraction at 313.15 $\mathrm{K}$, and Table 4 summarizes the MD results. Comparing this Figure with the VLE behaviour (see Figure 1 ), it is possible to observe that $\gamma$ is monotonously decreasing with mole fraction i.e. $\gamma$ decreases with the increase in concentration of the volatile component. Using a value $\chi_{i j}=0$ (see Eq. 7), SGT predicts $\gamma$ with an $\mathrm{AAD} \gamma$ of $1.10 \%$ with respect to the experimental data reported by Pugachevich and Belyarov [78]. This value is comparable to the results presented in a previous work [9] (AAD $\gamma$ of $1.20 \%$ ) and the results reported by Zuo and Stenby [23] whose used a SGT with Soave - Redlich - Kwong cubic EoS. In this latter work, Zuo and Stenby reported an AAD $\gamma$ of $1.00 \%$. We tested $\chi_{i j} \neq 0$ values but a 
marginal and non-statistically significant improvement of $\gamma$ predictions was found. At this point, it is important to note that with a value of $\chi_{i j}=0$ the SGT with SAFT model is a fully predictive scheme.

MD shows a good quantitative agreement to the experimental values with an AAD $\gamma$ of $1.79 \%$. We can conclude that both the theoretical approach (SGT with SAFT EoS) and MD simulation are capable of describing the bulk phase equilibrium and its interfacial tension with a relatively low AAD values.

Further to VLE and IFT, both theoretical and simulation approaches are able to describe the concentration profiles of species $\left(\rho_{i}\right)$ along the interface length $(z)$. For this mixture, Figures 4 show the $z-\rho_{i}$ projections as a function of the liquid molar fraction for some cases. Due to the symmetry of the vapour - liquid interfaces, and the periodical boundary condition used in MD, these Figures only include one vapour - liquid interface. In order to avoid the overlapping of $z-\rho_{i}$ projections, we displaced, for clarity, the localization of the bulk liquid and vapour phases. From the latter Figures, we observe that hexane exhibits a positive surface activity $\left(d \rho_{1} / d z=0 ; d^{2} \rho_{1} / d z^{2}<0\right.$ in the interfacial region), whereas decane does not show surface activity. The surface activity of hexane decreases as its mole fraction increases. In fact, according to our calculations, this maximum accumulation or absolute adsorption of hexane along the interfacial region corresponds to mole fraction of hexane in the vicinity of $0.40\left(x_{1} \approx 0.398\right)$. Additional to the surface activity patterns, Figures 4 show a widening of the interfacial regions as the mole fraction of hexane increases (or the mole fraction of decane decreases). The latter results agree to our previous finding on the effect of the molecular chain in the interfacial behaviour of mixtures [9].

Figure 5 shows the relative Gibbs adsorption isotherm of hexane (1) with respect to decane (2). In this Figure we include the results of $\left(\Gamma_{12}\right)$ from SGT (Eq. 3 and 4) and MD (Eq. 3). It is seen how $\Gamma_{12}$ increases as the liquid mole fraction of hexane increases without reaching a saturation limit. However, the magnitude of the relative Gibbs adsorption of hexane in decane is modest, as expected from inspection of Figures 4. Comparing the results from Eq. 3 and 4, it is possible to conclude that both approaches predict the same trends. Finally, comparing the MD results with SGT calculations, we conclude that MD shows a fairly good agreement to SGT, as expected since both results are based on $z$ $-\rho_{i}$ profiles, which are commensurate.

\subsection{Carbon dioxide + decane mixture}


There is an extensive set of data for this mixture, as it is prototypical in the oil and gas industry. Some of the most cited works are the experimental determinations of VLE and IFT reported for Nagarajan and Robinson [79] and Shaver et al., [80] at two isothermal conditions: $344.3 \mathrm{~K}$ and $337.6 \mathrm{~K}$, and the theoretical predictions from SGT with cubic like EoS [6, 21-24, 26] as well as SAFT EoS [32]. The reader is referred [26] for a complete review on IFT predictions for $\mathrm{CO}_{2}+$ hydrocarbon mixtures. While VLE as well as IFT of this mixture is well known, a comprehensive description of its interfacial properties and the corresponding MD simulations is still required. Since the main goal of this work is to gain insight in the capability to describe simultaneously the bulk and interfacial behaviour from theory, simulation and experimentation, we selected the isothermal condition of $344.3 \mathrm{~K}$ as a test condition.

Figures 6 and 7 shows VLE for this system $\left(\mathrm{CO}_{2}(1)+\right.$ decane (2)) at the quoted temperature. In these Figures, we have included the experimental results reported by Nagarajan and Robinson, SAFT calculations and MD results. Inspection of Figure 6 shows the extent of the positive deviations from ideal behaviour of this non-azeotropic mixture. As in the previous system, $k_{i j}$ of the SAFT-EoS was optimized using the available experimental information [79, 80] and constrained in order to avoid unstable liquid - liquid phase equilibria. The $k_{i j}$ value as well as deviation statistics for VLE predictions are summarized in Table 3.

From these results, we can observe that the SAFT model used in this work is reliable for correlating the VLE far from its critical state. In fact, our predictions show, far from its critical state, similar accuracy than other EoS models $[21,22,32]$. However, when the mixture approaches to its critical state, the EoS fails to predict accurately the VLE behaviour. Improved versions of SAFT, see for instance [81], overcome these deficiencies, common to the most analytical EoS.

Table 5 summarizes the MD results for this mixture. Comparing the MD results with SAFT calculations and experimental data, we observe that MD shows a good agreement to experimental data, with an AAD in vapour pressure of $4.5 \%$, and $\Delta y=0.404 \%$, which are slightly lower than the SAFT predictions.

In Figure 8 we show the interfacial tension for this mixture as a function of the liquid mole fraction at 344.3 K, and Table 5 summarizes the MD results. $\gamma$ has a negative slope on mole fraction; and $\gamma$ decreases as the concentration of $\mathrm{CO}_{2}$ (or the vapour pressure) increases (see Figure 6). As the mixture approaches the critical point, the accuracy of $\gamma$ predictions from SGT with SAFT decreases. A value of $\chi_{i j}=0$ in SGT accounted for an $\mathrm{AAD} \gamma$ equal to $13 \%$, with respect to the experimental data reported by Nagarajan and Robinson [79], which is similar than previous reported calculations [6, 21-24, 32]. This high value of $\mathrm{AAD} \gamma$ is caused by the inaccuracies of the SAFT model near to the critical state. In order to reduce the magnitude of $\mathrm{AAD} \gamma$, we tested other $\chi_{i j} \neq 0$ values but a non-statistically significant 
improvement of $\gamma$ predictions was found. In fact with a $\chi_{i j} \neq 0$ value, SGT deteriorates the estimation of $\gamma$, as Cornelisse [22] and Miqueu [36] pointed out. MD shows a good quantitative agreement to the experimental values with an $\mathrm{AAD} \gamma$ of $2.30 \%$. This value is lower than the results obtained from SGT. This difference is due to the fact that MD results are not so affected by the proximity of the critical state of the mixture.

Figure 9 shows the $z-\rho_{i}$ projections as a function of the liquid molar fraction. As with Figures 4, Figures 9 only include one vapour - liquid interface, and $z-\rho_{i}$ projections are displaced in order to avoid overlapping. From the latter Figures, we can observe $\mathrm{CO}_{2}$ exhibits a marked positive surface activity $\left(d \rho_{1} / d z=0 ; d^{2} \rho_{1} / d z^{2}<0\right.$ in the interfacial region), whereas decane does not show surface activity. As we can observe in Figure 9.a, the surface activity (or absolute adsorption) of $\mathrm{CO}_{2}$ increases as its liquid mole fraction increases. According to our calculations, this positive surface activity is present for the whole liquid mole fraction range of $\mathrm{CO}_{2}$, which is in agreement to the results reported by other authors (see Sahimi and Taylor [21], Lin et al. [26]). It is possible to observe from these Figures that MD results show similar concentration profiles along the interfacial region, although sub predicting of the absolute adsorption of $\mathrm{CO}_{2}$. This sub-prediction can be attributed to the deviations in the bulk concentrations, as observed in Figure 7.

Figure 10 shows the relative Gibbs adsorption isotherm of $\mathrm{CO}_{2}$ (1) with respect to decane (2) which is the component with the highest interfacial tension, and without absolute adsorption along the interfacial region (see Figure 9.b). As in the previous mixture, the relative Gibbs adsorption isotherm is calculated from SGT (Eq. 3 and 4) and MD results (Eq. 3). Figure 10 shows that this mixture exhibits a saturation limit (a maximum in $\Gamma_{12}$ ) and that the surface adsorption is significant for this mixture.

\subsection{Ethane + eicosane mixture}

This highly asymmetric mixture represents an extreme test case of a binary mixture composed of long n-alkane $\left(\mathrm{C}_{20}\right)$ in equilibria with a smaller solvent $\left(\mathrm{C}_{2}\right)$. The VLE behaviour of $\mathrm{C}_{2} \mathrm{H}_{6}(1)+\mathrm{C}_{20} \mathrm{H}_{42}(2)$ has been extensive measured by Peters et al., [82] at several isothermal conditions ranging from $270 \mathrm{~K}$ to $450 \mathrm{~K}$. In that work, Peters et al. also used the Soave - Redlich - Kwong EoS for correlating their experimental data. Following the results reported by Peters et al., we observe that a simple cubic EoS needs two mixture parameters (one for cohesion term and the other for the co-volume term) in order to model the high asymmetry of this mixture. Huang and Radosz [49] used it as test case of the SAFT EoS 
obtaining low values of AAD in vapour mole fraction with a single fitting. We are not aware of any reported interfacial tension properties of this mixture.

Figures 11 and 12 show VLE for this system at three different isothermal conditions $(300 \mathrm{~K}, 350 \mathrm{~K}$ and $400 \mathrm{~K})$. As with the other mixtures, these Figures include experimental results, SAFT calculations and MD simulations. The MD results exhibit a good agreement with the experimental information (see Table 6 for specific MD results). The total deviations of MD results obtained in this work are AADP = $2.80 \%$ and $\Delta y=0.156 \%$. As with the mixtures, Figure 12 shows an alternative VLE projection $(\rho-P$ diagram). In this Figure we can observe that SAFT and MD results exhibit an excellent agreement with each other.

Figure 13 shows the interfacial tension of this mixture as a function of the liquid mole fraction at 300 $\mathrm{K}, 350 \mathrm{~K}$ and $400 \mathrm{~K}$, and Table 6 summarizes the MD results. At isothermal conditions, $\gamma$ has negative slope on mole fraction, and for a fixed mole fraction, $\gamma$ decreases as the temperature increases.

SGT with a value of $\chi_{i j}=0$ is able to follow the MD results with a good agreement. Comparing the MD simulation to SGT prediction, we found an $\mathrm{AAD} \gamma$ equal to $5.56 \%$. Both the approaches are capable of describing bulk phase equilibrium and so it is sensible to assume that the same quality of fit is expected for the interfacial tension of this mixture at different isothermal conditions.

In figures 14 it is seen how the $z-\rho_{i}$ projections reflect an unusually high surface activity of ethane along the interfacial region. In fact, at fixed temperature, this mixture exhibits a strong positive surface activity for ethane whereas eicosane does not show surface activity. This absolute adsorption of ethane is always present for the whole mole fraction range and it increases as the liquid mole fraction of ethane increases.

The excess adsorption phenomena show similar patterns as the $\mathrm{CO}_{2}$ mixture. However, the most notorious variation of the absolute adsorption of ethane in eicosane is with temperature. For this system is possible to observe that, at fixed mole fraction, the absolute adsorption of ethane shows a strong increase as the temperature decreases. As an example of this behaviour, Figures 14 show the $z-\rho_{i}$ projections as a function of temperature at $x_{1} \approx 0.53$. Figure 15 is a snapshot of an equilibrium configuration of the mixture at the lower temperature. Here, the excess adsorption of ethane on the interfaces is seen clearly. A nanoscopic dense film is apparent on both of the bulk liquid surfaces.

Figures 16 shows the relative Gibbs adsorption isotherm of ethane (1) with respect to eicosane (2) which is the component with the highest interfacial tension, and without absolute adsorption along the interfacial region (see Figure 16.b). The relative Gibbs adsorption isotherm is calculated from SGT (Eq. 3 and 4) and MD results (Eq. 3). In Figure 16.a is possible to observe that $\Gamma_{12}$ increases as the 
liquid mole fraction of ethane increases without a saturation limit. This behaviour may be attributed to the proximity of a liquid - liquid - vapour (LLV) equilibria in this temperature range. According Peters et al. [83] this system exhibits a three phase line from 306.75 to $309.56 \mathrm{~K}$.

However, for the case of $350 \mathrm{~K}$ (Fig. 16.b) and $400 \mathrm{~K}$ (Fig. 16.c), we can observe that $\Gamma_{12}$ reaches a maximum value, which seems to correspond to the saturation limit for ethane, after which $\Gamma_{12}$ starts to decrease. Comparing the values of $\Gamma_{12}$ as a function of temperature, we can conclude that at fixed liquid mole fraction, $\Gamma_{12}$ decreases as the temperature increases

In complement to the previous interfacial properties predicted for this mixture, Figure 17 and Figure 18 show the surface entropy $\left(\Delta s^{\gamma}\right)$ and surface enthalpy $\left(\Delta h^{\gamma}\right)$ for this mixture, respectively, obtained from SGT and values of $\Delta s^{\gamma}$ and $\Delta h^{\gamma}$ at $350 \mathrm{~K}$ calculated from MD results, using a central differences scheme. From these Figures, it is possible to observe that both surface entropy (Fig. 17) and surface enthalpy (Fig. 18) decrease as the liquid mole fraction increases, and at fixed liquid mole fraction, these surface properties $\left(\Delta s^{\gamma}\right.$ and $\left.\Delta h^{\gamma}\right)$ decrease as the temperature increases. The behaviour observed in $\Delta s^{\gamma}$ and $\Delta h^{\gamma}$ can be rationalized by considering that at a fixed temperature, the increment of ethane (or reduction of eicosane) produces a more homogeneous interface and the energy required for interface formation decreases. At fixed liquid mole fraction, the interface is more homogenous and it needs less formation energy as the temperature increases. It is also possible that the vicinity of the LLV equilibria induces a high adsorption, previous to the appearance of a new liquid ethane phase.

\section{Concluding Remarks}

The main objective of this work is to simultaneously predict the phase equilibria and interfacial properties for hydrocarbon mixtures from Square Gradient Theory (SGT) and Molecular Dynamics (MD) simulations and to compare the results, when it possible, to available experimental data. The binary hydrocarbon mixtures considered in this work are all composed of a long n-alkane in equilibria with a smaller solvent: hexane + decane, carbon dioxide + decane, and ethane + eicosane.

In general terms, we can conclude that both the theoretical approach (SGT with SAFT EoS) and MD simulation are capable to describe the bulk phase equilibrium (VLE) and its interfacial tension (IFT) with a relatively low absolute average deviation values with respect to the experimental data. In addition to VLE and IFT, we show how it is possible to describe other interfacial properties such as concentration profiles along the interfacial region, surface activity (or absolute adsorption), and relative Gibbs adsorption isotherms for mixtures. In summary, we claim that both SGT and MD simulations are 
able to predict with an adequate agreement to each other the quoted interfacial properties for asymmetric binary hydrocarbon mixtures.

In all cases studied the interfacial tension exhibits a negative slope with mole fraction and decreases as the concentration of the volatile component (or vapour pressure of the system) increases. In addition to the interfacial tension behaviour, we record that the solvent exhibits a positive surface activity (or absolute adsorption) along the interfacial region; its surface activity is a function of the mole fraction. This positive absolute adsorption is attributed to the lower interfacial tension of the solvent, which implies its preference to locate itself in the interfacial region rather than the bulk phases. This behaviour is confirmed by the calculated relative Gibbs adsorption isotherms. These Gibbs adsorption isotherms exhibit two different patterns: For hexane + decane and ethane + eicosane at $300 \mathrm{~K}$, the relative Gibbs adsorption isotherm increases with the liquid mole fraction of solvent without reaching a saturation limit. A different pattern was detected for carbon dioxide + decane, and ethane + eicosane at $350 \mathrm{~K}$ and $400 \mathrm{~K}$, where a maximum relative Gibbs adsorption is evident at a certain liquid mole fraction of small solvent. The relative high values of the excess adsorption, sometimes leading to surface concentrations twice of those found in bulk regions, in these cases is noted.

\section{Acknowledgment}

Partial financial support from the Royal Society through the award of an International Short Visit grant VI 072256; from the U. K. Engineering and Physical Sciences Research Council through (EPSRC) grant EP/E016340 "Molecular Systems Engineering”, and FONDECYT, Santiago, Chile (Project 1080596) is gratefully acknowledged. The authors gratefully acknowledge the use of the GIBBS program suite, as part of a collaboration between Imperial College London and IFP, CNRS and Universite Paris Sud (Paris XI). 


\section{LIST OF SYMBOLS}

\begin{tabular}{|c|c|c|}
\hline$a$ & $=$ & Helmoltz energy density, A/V \\
\hline$A$ & $=$ & Helmholtz energy \\
\hline $\mathcal{A}$ & $=$ & interfacial area \\
\hline$c_{1}, c_{2}, c_{3}$ & $=$ & NERD constants in Eq. 15 \\
\hline$c_{i j}$ & $=$ & influence parameter \\
\hline$e$ & $=$ & constant in SAFT EoS \\
\hline$k_{B}$ & $=$ & Boltzmann's constant \\
\hline$k_{r}$ & $=$ & spring constant, Eq. 13 \\
\hline$k_{\theta}$ & $=$ & spring constant, Eq. 14 \\
\hline$k_{i j}$ & $=$ & interaction parameter for the EoS mixing rule \\
\hline$L_{x}, L_{y}, L_{z}$ & $=$ & cell dimension in $x, y, z$ \\
\hline$m$ & $=$ & molecular chain length \\
\hline$N$ & $=$ & Number of molecules \\
\hline$P$ & $=$ & absolute pressure \\
\hline$q_{i}$ & $=$ & atom charge \\
\hline$r_{i j}$ & $=$ & distance between united-atoms $i$ and $j$ \\
\hline$R$ & $=$ & universal gas constant \\
\hline$T$ & $=$ & absolute temperature \\
\hline$u_{i}^{o}$ & $=$ & temperature dependent well depth \\
\hline$U$ & $=$ & intermolecular potential \\
\hline$\Delta U$ & $=$ & change in configurational energy \\
\hline$v_{i}^{o o}$ & $=$ & segment volume \\
\hline$V$ & $=$ & volume \\
\hline$x, y$ & $=$ & mole fractions of the liquid and vapor phases \\
\hline$z$ & $=$ & coordinate normal to the interface \\
\hline$z_{0}^{\mathrm{j}}$ & $=$ & localization of the divide position relative to a species $j$ \\
\hline
\end{tabular}

\section{Greek}

$\alpha, \beta=$ bulk fluid phases in equilibrium 


$$
\begin{array}{ll}
\chi & =\text { adjustable parameter in Eq. } 7 \\
\Delta & =\text { Differential } \\
\Delta h^{\gamma} & =\text { enthalpy change of surface formation } \\
\Delta s^{\gamma} & =\text { entropy change of surface formation } \\
\Gamma_{i j} & =\text { Gibbs adsorption of a species } i \text { relatives to a species } j \\
\varepsilon & =\text { Lennard }- \text { Jones energy parameter } \\
\phi & =\text { dihedral angle between four consecutive united-atoms } \\
\gamma & =\text { interfacial tension } \\
\Lambda_{i} & \text { Broglie's wavelength } \\
\mu & =\text { chemical potential } \\
\theta & =\text { bond angle between three consecutive atoms } \\
\rho & =\text { molar concentration } \\
\sigma & =\text { Lennard }- \text { Jones size parameter } \\
\xi & =\text { magnitude of the perturbation }
\end{array}
$$

\section{Superscripts}

$$
\begin{array}{ll}
\text { chain } & =\text { chain contribution in SAFT }-\mathrm{EoS} \\
\text { disp } & =\text { dispersive contribution in SAFT }-\mathrm{EoS} \\
h s & =\text { repulsive contribution in SAFT }-\mathrm{EoS} \\
i g & =\text { ideal gas contribution in SAFT }-\mathrm{EoS} \\
R & =\text { residual contribution in SAFT }-\mathrm{EoS} \\
0 & =\text { equilibrium state }
\end{array}
$$

\section{Subscripts}

$0=$ equilibrium condition

$i, j=$ component $\mathrm{i}, \mathrm{j}$ respectively 


\section{REFERENCES}

1. G. Moriti, Oil and Gas Journal, 104 (2006) $37-41$.

2. M. Noh, A. Firoozabadi, SPE Reservoir Evaluation and Engineering. Aug. (2008) 676 - 685.

3. L. E. Urlic, L. J. Florusse, E. J. M. Straver, S. Degrange, C. J. Peters, Transp. Porous Media, 52 (2003) $141-157$.

4. T. Babadagli, J. Pet. Sci. Eng. 37 (2003) $25-37$.

5. V. S. Suicmez, M. Piri, M. J. Blunt, Adv Water Resour, 31 (2008) 503 - 512

6. A. Mejía, I. Polishuk, H. Segura, J. Wisniak. Thermochim. Acta, 411 (2004) 171 - 176.

7. A. Mejía, H. Segura, L. F. Vega, J. Wisniak. Fluid Phase Equilib. 227 (2005) 225 - 238.

8. A. Mejía, H. Segura, J. Wisniak, I. Polishuk, J. Phase Equilib. Diffus. 26 (2005) 1 - 9.

9. A. Mejía, H. Segura, J. Wisniak, I. Polishuk. Phys. Chem. Liq. 44 (2006) 45 - 59.

10. A. Mejía, J. C. Pàmies, D. Duque, H. Segura, L. F. Vega, J. Chem. Phys. 123 (2005) 034505 034515

11. A. Mejía, L. F. Vega, J. Chem. Phys. 124 (2006) 244505-1 - 244505-7

12. P. Fertl. Molecular Simulation of hydrocarbon mixtures. MSc. Thesis, Imperial College (2006)

13. V. L. Quinn, Molecular Simulation of hydrocarbon fluids MSc. Thesis, Imperial College (2006)

14. D. B. MacLeod, Trans. Faraday Soc. 19 (1923) 38-43, for a recent review see Chap. 12 in The

Properties of Gases and Liquids, 5th ed, by B. E. Poling, J. M. Prausnitz, J. O'Connell, McGraw-Hill, 2002

15. V. Bongiorno, H.T. Davis, Phys. Rev. A 12 (1975) 2213 - 2224.

16. R. Evans, Adv. Phys. 28 (1979) 143-200.

17. S. Jain, A. Dominik, W. G. Chapman, J. Chem. Phys. 127 (2007) 244904-1 - 244904-12.

18. J. D. van der Waals, Zeit. Phys. Chem. 13 (1893) 657-725 (see J. S. Rowlinson, J. Statist. Phys. 20 (1979) 197-244 for an English translation)

19. J. W. Cahn, J. E. Hilliard, J. Chem. Phys. 28 (1958) 258 - 267.

20. B. S. Carey, L. E. Scriven, H. T. Davis, AIChE J. 24 (1978) 1076 - 1080; ibid, AIChE J. 26 (1980) $705-711$.

21. M. Sahimi, B. Taylor, J. Chem. Phys. 95 (1991) 6749 - 6761.

22. P. M. W. Cornelisse, C. J. Peters, J. de Swaan Arons, Fluid Phase Equilib. 82 (1993) 119 - 129; ibid Mol. Phys. 80 (1993) 941 - 955; ibid Fluid Phase Equilib. 117 (1996) 312 - 319.

23. Y. X. Zuo, E. H. Stenby, Fluid Phase Equilib. 132 (1997) 139 - 158. 
24. C. Miqueu, B. Mendiboure, A. Graciaa and J. Lachaise, Fluid Phase Equilib. 207 (2003) 225 - 246; ibid, Fluid Phase Equilib. 218 (2004) 189 - 203; ibid, Ind. Eng. Chem. Res. 44 (2005) 3321 - 3329.

25. A. J. Queimada, C. Miqueu, I. M. Marrucho, G. M. Kontogeorgis, J. A. P. Coutinho, Fluid Phase Equilib. 228 - 229 (2005) 479 - 485.

26. H. Lin, Y-Y. Duan, Q. Mina, Fluid Phase Equilib. 254 (2007) 75 - 90.

27. M.B. Oliveira, I.M. Marrucho, J.A.P. Coutinho, A.J. Queimada, Fluid Phase Equilib. 267 (2008) 83 -91 .

28. P. M. W. Cornelisse, M. Wijtkamp, C. J. Peters, J. de Swaan Arons, Fluid Phase Equilib. 150 (1998) $633-640$.

29. S. Enders, H. Kahl, Fluid Phase Equilib. 172 (2000) 27 - 42.

30. H. Kahl, S. Enders, Phys. Chem. Chem. Phys. 4 (2002) $931-936$.

31. D. Fu, X. S Li, S. M. Yan, T. Liao, Ind. Eng. Chem. Res. 45 (2006) 8199 - 8206.

32. D. Fu, Y. Wei, Ind. Eng. Chem. Res. 47 (2008) 4490 - 4495.

33. D. Fu, H. J. Jiang, B.S. Wang, Fluid Phase Equilib. 279 (2009) 136-140.

34. B. S. Carey, The Gradient Theory of Fluid Interfaces, Ph.D. Thesis, University of Minnesota, 1979.

35. P. M. W. Cornelisse, The Gradient Theory Applied, Simultaneous Modelling of Interfacial Tension and Phase Behaviour, Ph.D. Thesis, Delft University, 1997.

36. C. Miqueu, Modélisation, à température et pression élevées, de la tension superficielle de composants des fluides pétroliers et de leurs mélanges synthétiques ou réels, Ph.D. Thesis, Université de Pau et des Pays de 1'Adour, France, 2001.

37. A. Mejía, Comportamiento Interfacial de Mezclas Fluidas en Equilibrio, Ph. D. Thesis, Universidad de Concepción, Chile, 2004.

38. A. Mejía, H. Segura. Int. J. Thermophys. 25 (2004) 1395 - 1445.

39. Y. S. Wei, R. Sadus, AIChE J. 46 (2000) 169 - 196.

40. E. A. Müller, K. E. Gubbins, In Equations of State for Fluids and Fluid Mixtures; J. V. Sengers, R. F. Kayser, C. J. Peters, H. J. Jr. White, Eds.; Elsevier, New York (2000) 435 - 477.

41. E. A. Müller, K. E. Gubbins, Ind. Eng. Chem. Res. 40 (2001) 2193 - 2211.

42. I. G. Economou, Ind. Eng. Chem. Res. 41 (2002) 953 - 962.

43. P. Paricaud, A. Galindo, G. Jackson, Fluid Phase Equilib. 194-197 (2002) 87 - 96.

44. S. P. Tan, H. Adidharma, M. Radosz, Ind. Eng. Chem. Res. 47 (2008) 8063 - 8082.

45. J. S. Rowlinson and B. Widom, Molecular Theory of Capillarity, Oxford University Press, Oxford, 1989.

46. R. Defay, I. Prigogine, Surface Tension and Adsorption, Longmans, London 1966. 
47. H. Kahl, T. Wadewitz, J. Winkelmann, J. Chem. Eng. Data. 48 (2003) 580 - 586.

48. J. W. Tester, M. Modell, Thermodynamics and its Applications. 3rd edition, Prentice Hall, New Jersey, 1997 .

49. S. H. Huang, M. Radosz, Ind. Eng. Chem. Res. 29 (1990) 2284 - 2294. ; ibid, Ind. Eng. Chem. Res. 30 (1990) $1994-2005$.

50. J. M. Prausnitz, R. N. Lichtenthaler, E. Gomes de Azevedo, Molecular Thermodynamics of FluidPhase Equilibria, 3th ed., Prentice-Hall, New Jersey, 1999.

51. F. Martinez-Veracoechea, E. A. Müller, Mol. Simulat. 31 (2005) 33 - 43.

52. K. Binder, Phys. Rev. A 25 (1982) 1699 - 1709.

53. J. J. Potoff, A. Z. Panagiotopoulos, J. Chem. Phys., 112 (2000) $6411-6415$.

54. J. I. Siepmann, D. Frenkel, Mol. Phys., 75 (1992) 59 - 70.

55. D. Frenkel, G.C.A.M. Mooij, B. Smit, J. Phys.:Condns. Matter, 4 (1992) 3053 - 3076.

56. J. J. de Pablo, M. Laso, U.W. Suter, J. Chem. Phys., 96 (1992) 2395 - 2403.

57. S. K. Nath, F. A. Escobedo, J. J. dePablo, J. Chem. Phys. 108 (1998) 9905 - 9911.

58. W. L. Jorgensen, J. D. Madura, C. J. Swenson, J. Am. Chem. Soc. 106 (1984) 6638 - 6646.

59. J. G. Harris, K. H. Yung, J. Phys. Chem. 99 (1986) 12021 - 12024.

60. C. D. Holcomb, P. Clancy, J. A. Zollweg, Mol. Phys. 78 (1993) 437 - 459.

61.A. Trokhymchuk, J. Alejandre, J. Chem. Phys. 111 (1999) $8510-8523$.

62. D. Duque, L. F. Vega, J. Chem. Phys. 121 (2004) 8611 - 8617.

63. F. J. Blas, L. G. MacDowell, E. de Miguel, G. Jackson, J. Chem. Phys. 129 (2008) 144703-1 144703-9.

64. M. Gonzalez-Melchor, P. Orea, J. Lopez-Lemus, F. Bresme, J. Alejandre, J. Chem. Phys. 122 (2005) 094503-1 - 094503-8.

65. DL_POLY Molecular Simulation Package, http://www.ccp5.ac.uk/DL_POLY/. See also W. Smith, T. R. Forester, Journal of Molecular Graphics 14 (1996) 136.

66. GIBBS Program suite, property of the Institut Francais du Petrole (IFP), CNRS and Universite Paris Sud (Paris XI).

67. D. Frenkel. B. Smit, Understanding Molecular Simulation. 2 edition, Academic Press, 2002.

68 G. J. Gloor, G. Jackson, F. J. Blas, E. de Miguel, J. Chem. Phys. 123 (2005) 134703-1 - 134703-19.

69. J. R. Errington, D. A. Kofke, J. Chem. Phys. 127 (2007) 174709-1 - 174709-12.

70. A. Ghoufi, F. Goujon, V. Lachet, P. Malfreyt, J. Chem. Phys. 128 (2008) 154716-1 - 154716-15.

71. E. de Miguel, G. Jackson, J. Chem. Phys., 125 (2005) 164109-1 - 164109-11. 
72. C. Ibergay, A. Ghoufi, F. Goujon, P. Ungerer, A. Boutin, B. Rousseau, P. Malfreyt, Phys. Rev. E. 75 (2007) 051602-1 - 051602-18.

73. A. Ghoufi, F. Goujon, V. Lachet, P. Malfreyt, J. Chem. Phys. 128 (2008) 154716-1 - 154716-15.

74. F. Biscay, A. Ghoufi, F. Goujon, V. Lachet, P. Malfreyt, J. Phys. Chem. B 112 (2008) 13885 13897.

75. F. J. Blas, L. G. MacDowell, E. de Miguel, G. Jackson, J. Chem. Phys. 129 (2008) 144703-1 144703-9.

76. Wohlfarth, Ch and B. Wohlfarth, Numerical Data and Functional Relationships in Science and Technology, Vol. 16, Surface Tension of Pure Liquids and Binary Liquid Mixtures, Eds. Lechner, M. D. in Landolt-Börnstein, New Series Group IV Physical Chemistry (Springer Verlag Berlin, Heidelberg, 1997)

77. K. N. Marsh, J. B. Ott, M. J. Costigan, J. Chem. Thermodyn. 12 (1980) 343 - 348.

78. P. P. Pugachevich, E. M. Belyarov, Kolloidn. Zh. 32 (1970) 895.

79. N. Nagarajan, R. L. Jr. Robinson J. Chem. Eng. Data, 31 (1986) 168 - 171.

80 R. D. Shaver, R. L. Robinson Jr., K. A. M. Gasem, Fluid Phase Equilib. 179 (2000) 43 - 66.

81. L. Sun, H. Zhao, S. B. Kiselev, C. McCabe, J. Phys. Chem. B 109 (2005) 9047 - 9058; ibid, Fluid Phase Equilib. 228-229 (2005) 275-282.

82. C. J. Peters, J. L. de Roo, R. N. Lichtenthaler, Fluid Phase Equilib. 34 (1987) 287 - 308.

83. C. J. Peters, J. L. de Roo, R. N. Lichtenthaler, Fluid Phase Equilib. 69 (1991) 51 - 66. 
Table 1. Box dimensions and cut-off radius used in MD simulations

\begin{tabular}{lccc}
\hline \multicolumn{1}{c}{ mixture } & $\boldsymbol{L}_{\boldsymbol{x}}=\boldsymbol{L}_{\boldsymbol{y}} / \boldsymbol{A}$ & $\boldsymbol{L}_{z} / \boldsymbol{A}$ & $\boldsymbol{r}_{\boldsymbol{c}} / \boldsymbol{A}$ \\
\hline hexane (1) + decane (2) & 52 & 156 & 20.0 \\
carbon dioxide (1) + decane (2) & 52 & 156 & 20.0 \\
ethane (1) + eicosane (2) & 50 & 150 & 13.8 \\
\hline
\end{tabular}


Table 2. Thermophysical properties of the pure components ${ }^{\mathrm{a}}$

\begin{tabular}{|c|c|c|c|c|}
\hline Fluid & $m_{i}$ & $\begin{array}{c}v_{i}^{o o} \\
(m L / m o l)\end{array}$ & $\begin{array}{c}\boldsymbol{u}_{i}^{o} / \boldsymbol{k}_{B} \\
(K)\end{array}$ & $\begin{array}{c}10^{20} \times c_{i i}{ }^{0} / \\
\left(J m^{5} m o I^{2}\right)\end{array}$ \\
\hline eicosane & 13.940 & 12.000 & 211.25 & 358.6851 \\
\hline ethane & 1.941 & 14.460 & 191.44 & 4.9632 \\
\hline carbon dioxide & 1.417 & 13.578 & 216.08 & 2.0039 \\
\hline decane & 7.527 & 11.723 & 205.46 & 82.5242 \\
\hline hexane & 4.724 & 12.475 & 202.72 & 35.6556 \\
\hline
\end{tabular}

${ }^{a}$ Pure fluid parameters of the SAFT-EoS have been taken from Huang and Radosz [49]. $c_{i i}$ are fitted from experimental $\gamma$ data, taken from Landolt-Börnstein data base [76] 
Table 3. Binary parameters for mixing rules and statistic deviations in vapour pressure and vapour phase mole fractions for VLE correlations and interfacial tensions predictions

\begin{tabular}{|c|c|c|c|c|c|}
\hline System & $T / K$ & $\boldsymbol{k}_{i j}^{a}$ & $A A D P \%^{b}$ & $\Delta y_{1}^{c}$ & $A A D \gamma \%^{b}$ \\
\hline \multirow[t]{2}{*}{ hexane $(1)+$ decane $(2)$} & 308.15 & -0.0013 & 2.0 & 0.050 & \\
\hline & 313.15 & --- & --- & --- & 1.1 \\
\hline \multirow[t]{2}{*}{$(1)+$ decane $(2)$} & 344.30 & 0.1350 & 3.9 & 1.462 & 13.0 \\
\hline & 300.00 & & 1.7 & 0.001 & --- \\
\hline \multirow[t]{2}{*}{ ethane (1) + eicosane (2) } & 350.00 & 0.0300 & 5.3 & 0.395 & --- \\
\hline & 400.00 & & 6.6 & 0.197 & --- \\
\hline
\end{tabular}

${ }^{a}$ The $k_{i j}$ parameters are fitted from experimental VLE data, taken from Marsh et al. [77] (hexane + decane); Nagarajan and Robinson [79] and ] ( + decane); Peters et al. [82] (ethane + eicosane). ${ }^{\mathrm{b}} \mathrm{AAD}$ is the absolute average deviation, AAD $\delta=$ $\left(100 / \mathrm{N}_{\mathrm{P}}\right) \sum_{\mathrm{i}=1, \mathrm{NP}}\left|\delta_{\mathrm{i}}{ }^{\mathrm{exp}}-\delta_{\mathrm{i}}^{\mathrm{cal}}\right| / \delta_{\mathrm{i}}^{\mathrm{exp}}(\delta=\mathrm{P}$, or $\gamma){ }^{\mathrm{c}} \Delta \mathrm{y}_{1}=\left(100 / \mathrm{N}_{\mathrm{P}}\right) \times \sum_{\mathrm{i}=1, \mathrm{NP}}\left|\mathrm{y}_{\mathrm{i}}{ }^{\text {exp }}-\mathrm{y}_{\mathrm{i}}^{\mathrm{cal}}\right| . \mathrm{N}_{\mathrm{P}}$ is the number of experimental points. VLE deviations are measured with respect to the experimental data used for $k_{i j}$ and the $\gamma$ deviations are measured respect to the experimental $\gamma$ data, taken from Landolt-Börnstein data base [76]. 
Table 4. Phase equilibria and interfacial tension results from Molecular Dynamics for hexane (1) + decane (2) mixture at $313.15 \mathrm{~K}^{\mathrm{a}}$

\begin{tabular}{cccccccc}
\hline$N_{l}$ & $N_{2}$ & $\begin{array}{c}P \\
(\mathrm{kPa})\end{array}$ & $\begin{array}{c}\rho_{L} \\
\left(\mathrm{kmol} \mathrm{m}^{-3}\right)\end{array}$ & $\begin{array}{c}\rho_{V} \\
\left(\mathrm{kmol} \mathrm{m}^{-3}\right)\end{array}$ & & $y_{1}$ & $\begin{array}{c}\gamma \\
\left(\mathrm{mN} \mathrm{m}^{-1}\right)\end{array}$ \\
\hline 0 & 448 & $0.457_{5}$ & $5.192_{2}$ & $0.004_{6}$ & $0.000_{0}$ & $0.000_{0}$ & $21.90_{1}$ \\
120 & 360 & $9.689_{9}$ & $5.529_{3}$ & $0.0050_{4}$ & $0.229_{1}$ & $0.970_{3}$ & $20.62_{6}$ \\
280 & 280 & $19.716_{4}$ & $6.009_{8}$ & $0.0112_{7}$ & $0.480_{2}$ & $0.979_{8}$ & $19.07_{5}$ \\
420 & 120 & $30.787_{4}$ & $6.791_{1}$ & $0.0195_{7}$ & $0.764_{1}$ & $0.989_{8}$ & $17.47_{3}$ \\
720 & 0 & $38.339_{2}$ & $7.493_{6}$ & $0.0241_{2}$ & $1.000_{0}$ & $1.000_{0}$ & $16.24_{1}$ \\
\hline
\end{tabular}

${ }^{a}$ The subscripted number is the uncertainty in the last digit. (i.e. $0.457_{5}$ means $0.457 \pm 0.0005$ ) 
Table 5. Phase equilibria and interfacial tension results from Molecular Dynamics for $\mathrm{CO}_{2}(1)+$ decane (2) at $344.3 \mathrm{~K}^{\mathrm{a}}$

\begin{tabular}{cccccccc}
\hline$N_{I}$ & $N_{2}$ & $\begin{array}{c}P \\
(\mathrm{MPa})\end{array}$ & $\begin{array}{c}\rho_{L} \\
\left(\mathrm{kmol} \mathrm{m}^{-3}\right)\end{array}$ & $\begin{array}{c}\rho_{V} \\
\left(\mathrm{kmol} \mathrm{m}^{-3}\right)\end{array}$ & & $y_{1}$ & $\begin{array}{c}\gamma \\
\left(\mathrm{mN} \mathrm{m}^{-1}\right)\end{array}$ \\
\hline 0 & 448 & $0.121_{1}$ & $5.177_{2}$ & $0.070_{8}$ & $0.000_{0}$ & $0.000_{0}$ & $18.93_{1}$ \\
235 & 400 & $2.653_{2}$ & $5.927_{3}$ & $0.574_{4}$ & $0.174_{2}$ & $0.993_{4}$ & $15.20_{5}$ \\
352 & 300 & $4.586_{8}$ & $6.658_{4}$ & $1.212_{9}$ & $0.320_{5}$ & $0.994_{4}$ & $11.90_{3}$ \\
504 & 252 & $6.679_{2}$ & $7.763_{2}$ & $2.023_{5}$ & $0.448_{1}$ & $0.996_{9}$ & $8.46_{3}$ \\
648 & 216 & $7.808_{7}$ & $8.290_{9}$ & $2.622_{8}$ & $0.512_{4}$ & $0.997_{3}$ & $6.69_{4}$ \\
800 & 200 & $9.780_{9}$ & $9.692_{3}$ & $4.408_{6}$ & $0.633_{6}$ & $0.998_{6}$ & $3.67_{7}$ \\
875 & 125 & $11.384_{2}$ & $11.106_{8}$ & $6.367_{1}$ & $0.751_{2}$ & $0.998_{7}$ & $1.26_{5}$ \\
\hline
\end{tabular}

${ }^{a}$ The subscripted number is the uncertainty in the last digit. (i.e. $6.679_{2}$ means $6.679 \pm 0.0002$ ) 
Table 6. Phase equilibria and interfacial tension results from Molecular Dynamics for ethane (1) + eicosane (2) mixture ${ }^{\mathrm{a}}$

$$
\mathrm{T}=300 \mathrm{~K}
$$

\begin{tabular}{|c|c|c|c|c|c|c|c|}
\hline$N_{l}$ & $N_{2}$ & $\begin{array}{c}P \\
(\mathrm{MPa})\end{array}$ & $\begin{array}{c}\rho_{L} \\
\left(\mathrm{kmol} \mathrm{m}^{-3}\right)\end{array}$ & $\begin{array}{c}\rho_{V} \\
\left(\mathrm{kmol} \mathrm{m}^{-3}\right)\end{array}$ & $x_{1}$ & $y_{1}$ & $\begin{array}{c}\gamma \\
\left(\mathrm{mN} \mathrm{m}^{-1}\right)\end{array}$ \\
\hline 398 & 260 & $0.907_{1}$ & $3.368_{6}$ & $0.389_{5}$ & $0.229_{3}$ & $0.999_{1}$ & $24.45_{9}$ \\
\hline 615 & 238 & $1.697_{7}$ & $4.350_{6}$ & $0.862_{7}$ & $0.410_{4}$ & $0.999_{5}$ & $18.78_{9}$ \\
\hline 752 & 225 & $2.216_{6}$ & $4.995_{6}$ & $1.207_{7}$ & $0.532_{5}$ & $0.999_{7}$ & $14.94_{8}$ \\
\hline 890 & 211 & $2.804_{9}$ & $5.689_{3}$ & $1.391_{5}$ & $0.639_{3}$ & $0.999_{3}$ & $10.79_{5}$ \\
\hline 1000 & 200 & $3.438_{2}$ & $6.843_{6}$ & $2.084_{7}$ & $0.730_{9}$ & $0.998_{8}$ & $7.25_{7}$ \\
\hline
\end{tabular}

$$
\mathrm{T}=350 \mathrm{~K}
$$

\begin{tabular}{|c|c|c|c|c|c|c|c|}
\hline$N_{l}$ & $N_{2}$ & $\begin{array}{c}P \\
(\mathrm{MPa})\end{array}$ & $\begin{array}{c}\rho_{L} \\
\left(\mathrm{kmol} \mathrm{m}^{-3}\right)\end{array}$ & $\begin{array}{c}\rho_{V} \\
\left(\mathrm{kmol} \mathrm{m}^{-3}\right)\end{array}$ & $x_{1}$ & $y_{1}$ & $\begin{array}{c}\gamma \\
\left(\mathrm{mN} \mathrm{m}^{-1}\right)\end{array}$ \\
\hline 407 & 259 & $1.331_{1}$ & $3.191_{7}$ & $0.697_{9}$ & $0.238_{4}$ & $0.999_{8}$ & $19.59_{5}$ \\
\hline 623 & 238 & $2.678_{5}$ & $4.098_{6}$ & $1.290_{8}$ & $0.418_{5}$ & $0.999_{6}$ & $15.03_{3}$ \\
\hline 760 & 224 & $3.833_{4}$ & $4.792_{2}$ & $1.783_{8}$ & $0.532_{5}$ & $0.999_{4}$ & $11.46_{3}$ \\
\hline 1000 & 200 & $6.658_{2}$ & $6.501_{5}$ & $3.668_{5}$ & $0.732_{7}$ & $0.998_{5}$ & $5.21_{9}$ \\
\hline 1132 & 187 & $9.630_{8}$ & $8.254_{5}$ & $7.568_{8}$ & $0.843_{1}$ & $0.998_{7}$ & $1.77_{6}$ \\
\hline
\end{tabular}

$$
\mathrm{T}=400 \mathrm{~K}
$$

\begin{tabular}{cccccccc}
\hline$N_{1}$ & $N_{2}$ & $\begin{array}{c}P \\
(\mathrm{MPa})\end{array}$ & $\begin{array}{c}\rho_{L} \\
\left(\mathrm{kmol} \mathrm{m}^{-3}\right)\end{array}$ & $\begin{array}{c}\rho_{V} \\
\left(\mathrm{kmol} \mathrm{m}^{-3}\right)\end{array}$ & $\begin{array}{c}x_{1} \\
y_{1}\end{array}$ & $\begin{array}{c}\gamma \\
\left(\mathrm{mN} \mathrm{m}^{-1}\right)\end{array}$ \\
\hline 412 & 259 & $1.932_{9}$ & $3.008_{7}$ & $0.473_{5}$ & $0.230_{3}$ & $0.997_{3}$ & $15.58_{2}$ \\
632 & 237 & $3.970_{3}$ & $3.613_{4}$ & $1.148_{8}$ & $0.414_{2}$ & $0.996_{9}$ & $12.04_{1}$ \\
774 & 223 & $5.719_{3}$ & $4.303_{7}$ & $1.987_{9}$ & $0.532_{5}$ & $0.997_{7}$ & $9.20_{5}$ \\
1000 & 200 & $10.235_{1}$ & $5.837_{1}$ & $4.008_{2}$ & $0.720_{5}$ & $0.997_{4}$ & $3.85_{6}$ \\
1156 & 184 & $15.857_{9}$ & $7.864_{4}$ & $7.183_{5}$ & 0.8506 & $0.997_{3}$ & $1.02_{4}$ \\
\hline
\end{tabular}

\footnotetext{
${ }^{\mathrm{a}}$ The subscripted number is the uncertainty in the last digit. (i.e. $0.907_{1}$ means $0.907 \pm 0.0001$ )
} 


\section{Figure Captions}

[1] Isothermal vapour - liquid equilibria for hexane (1) + decane (2) mixture. ( $\bigcirc)$ Experimental data at $308.15 \mathrm{~K}$ [77]; (- -) SAFT - EoS at 308.15 K; (-) SAFT -EoS at $313.15 \mathrm{~K}$; (*) MD results at $313.15 \mathrm{~K}$.

[2] $\quad P-\rho$ diagram for hexane (1) + decane (2) mixture at $313.15 \mathrm{~K} .(-) \mathrm{SAFT}-\mathrm{EoS}$; (*) MD results.

[3] Interfacial tension for hexane (1) + decane (2) mixture at 313.15 K. (O) Experimental data [78]; (一) SGT + SAFT - EoS; (*) MD results.

[4] (a) $z-\rho_{l}$, (b) $z-\rho_{2}$ profiles for hexane (1) + decane (2) mixture at $313.15 \mathrm{~K}$. (-) SGT + SAFT - EoS; (*) MD results.

[5] Relative Gibbs adsorption isotherm for hexane (1) + decane (2) mixture at $313.15 \mathrm{~K}$ as a function of the liquid mole fraction. (-) Eq. 3; (--) Eq. 4; (*) MD results.

[6] Vapour - liquid equilibria for $\mathrm{CO}_{2}(1)+$ decane (2) mixture at $344.3 \mathrm{~K}$. (O) Experimental data [79]; (-) SAFT -EoS; (*) MD results.

[7] $\quad P-\rho$ diagram for $\mathrm{CO}_{2}(1)+$ decane (2) mixture at $344.3 \mathrm{~K}$. (O) Experimental data [79]; (一)SAFT - EoS; (*) MD results.

[8] Interfacial tension for $\mathrm{CO}_{2}(1)+$ decane (2) mixture at $344.3 \mathrm{~K}$. (O) Experimental data [79]; (一) $\mathrm{SGT}+\mathrm{SAFT}-\mathrm{EoS} ;($ *) MD results.

[9] (a) $z-\rho_{1}$, (b) $z-\rho_{2}$ profiles for $\mathrm{CO}_{2}(1)+$ decane (2) mixture at $344.3 \mathrm{~K}$. (-) SGT + SAFT EoS; (*) MD results.

[10] Relative Gibbs adsorption isotherm for $\mathrm{CO}_{2}(1)+$ decane (2) mixture at $344.3 \mathrm{~K}$ as a function of the liquid mole fraction. (-) Eq. 3; (- -) Eq. 4; (*) MD results.

[11] Isothermal vapour - liquid equilibria for ethane (1) + eicosane (2) mixture. (O) Experimental data [82]; (-) SAFT -EoS; MD results at (+) $300 \mathrm{~K},(\times) 350 \mathrm{~K},(*) 400 \mathrm{~K}$. 
[12] $P-\rho$ diagram for ethane (1) + eicosane (2) mixture. (-)SAFT - EoS; MD results at (+) $300 \mathrm{~K}$, (×) $350 \mathrm{~K},(*) 400 \mathrm{~K}$.

[13] Interfacial tension for ethane (1) + eicosane (2) mixture. (-) SGT + SAFT - EoS; MD results at (+) $300 \mathrm{~K},(\times) 350 \mathrm{~K},(*) 400 \mathrm{~K}$.

[14] (a) $z-\rho_{1}$, (b) $z-\rho_{2}$ profiles for ethane (1) + eicosane (2) mixture at $x_{1} \approx 0.53$ and $300 \mathrm{~K}, 350 \mathrm{~K}$, and $400 \mathrm{~K}$. (-) SGT + SAFT - EoS; (*) MD results. Middle (unlabeled curves) are at 350K.

[15] Snapshot of an equilibrium configuration for ethane (red or gray ) + eicosane (white or light) mixture at $\mathrm{T}=300 \mathrm{~K}$, and at $x_{1} \approx 0.731$.

[16] Relative Gibbs adsorption isotherm for ethane (1) + eicosane (2) mixture as a function of the liquid mole fraction. (-)Eq. 3; (--) Eq. 4; (*) MD results.

a. $\mathrm{T}=300 \mathrm{~K}, \mathrm{~b} . \mathrm{T}=350 \mathrm{~K}, \mathrm{c} . \mathrm{T}=400 \mathrm{~K}$

[17] Variation of surface entropy with the liquid mole fraction for ethane (1) + eicosane (2) mixture at $300 \mathrm{~K}, 350 \mathrm{~K}$ and $400 \mathrm{~K}$. (-) SGT calculations; (*) MD results at $350 \mathrm{~K}$.

[18] Variation of surface enthalpy with the liquid mole fraction for ethane (1) + eicosane (2) mixture at $300 \mathrm{~K}, 350 \mathrm{~K}$ and $400 \mathrm{~K}$. (-) SGT calculations; (*) MD results at $350 \mathrm{~K}$. 


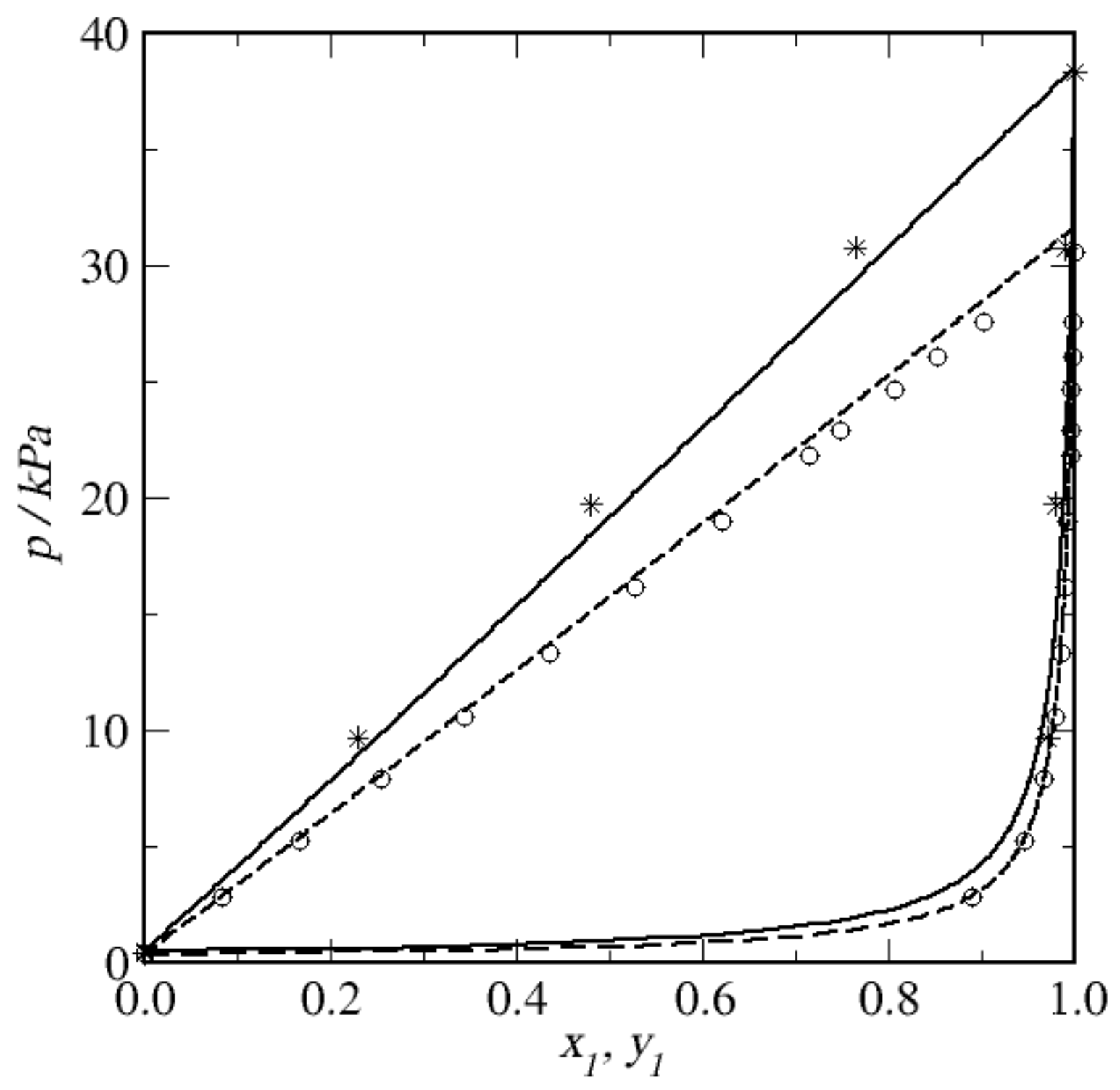

Figure 1 


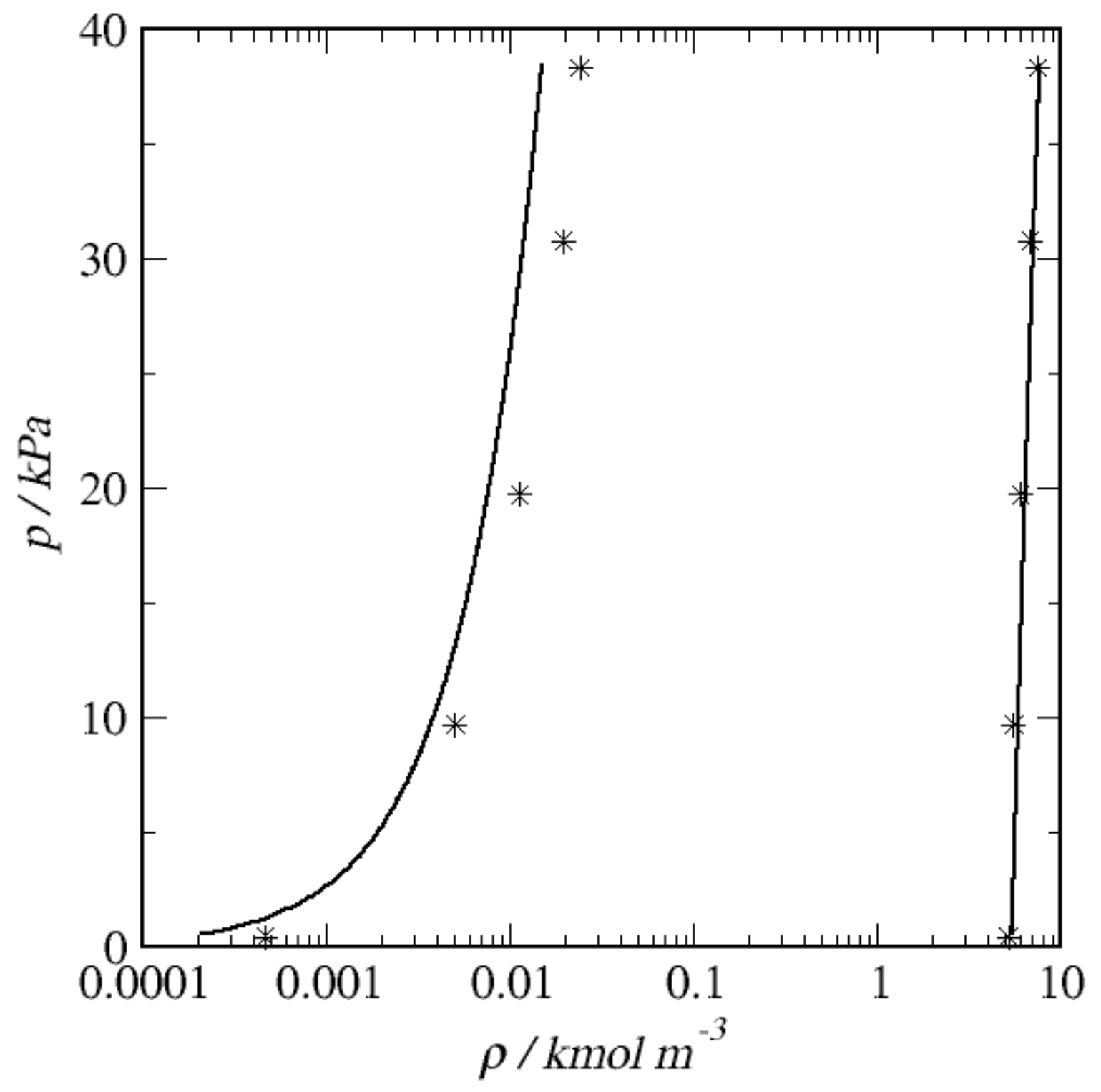

Figure 2 


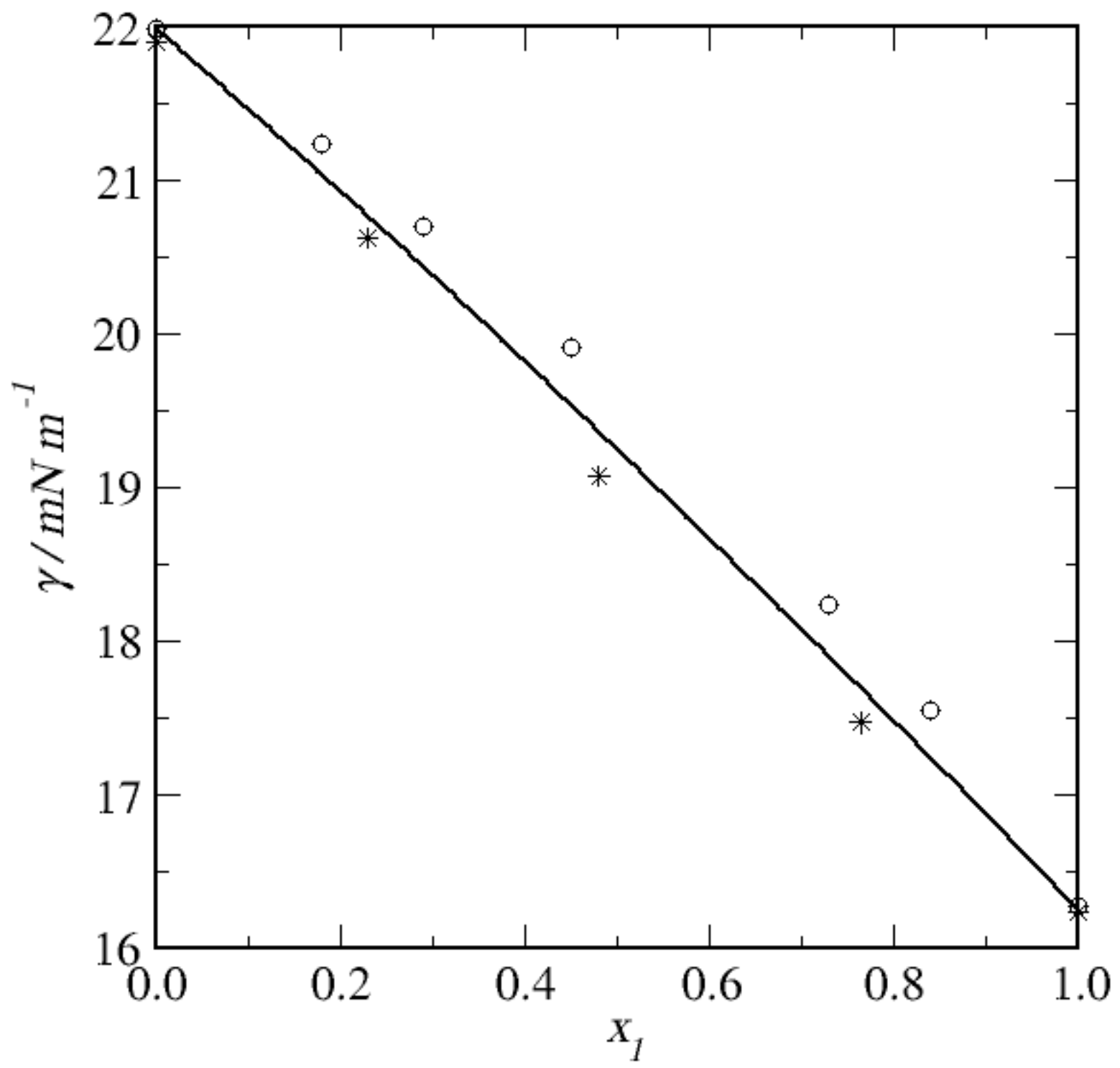

Figure 3 


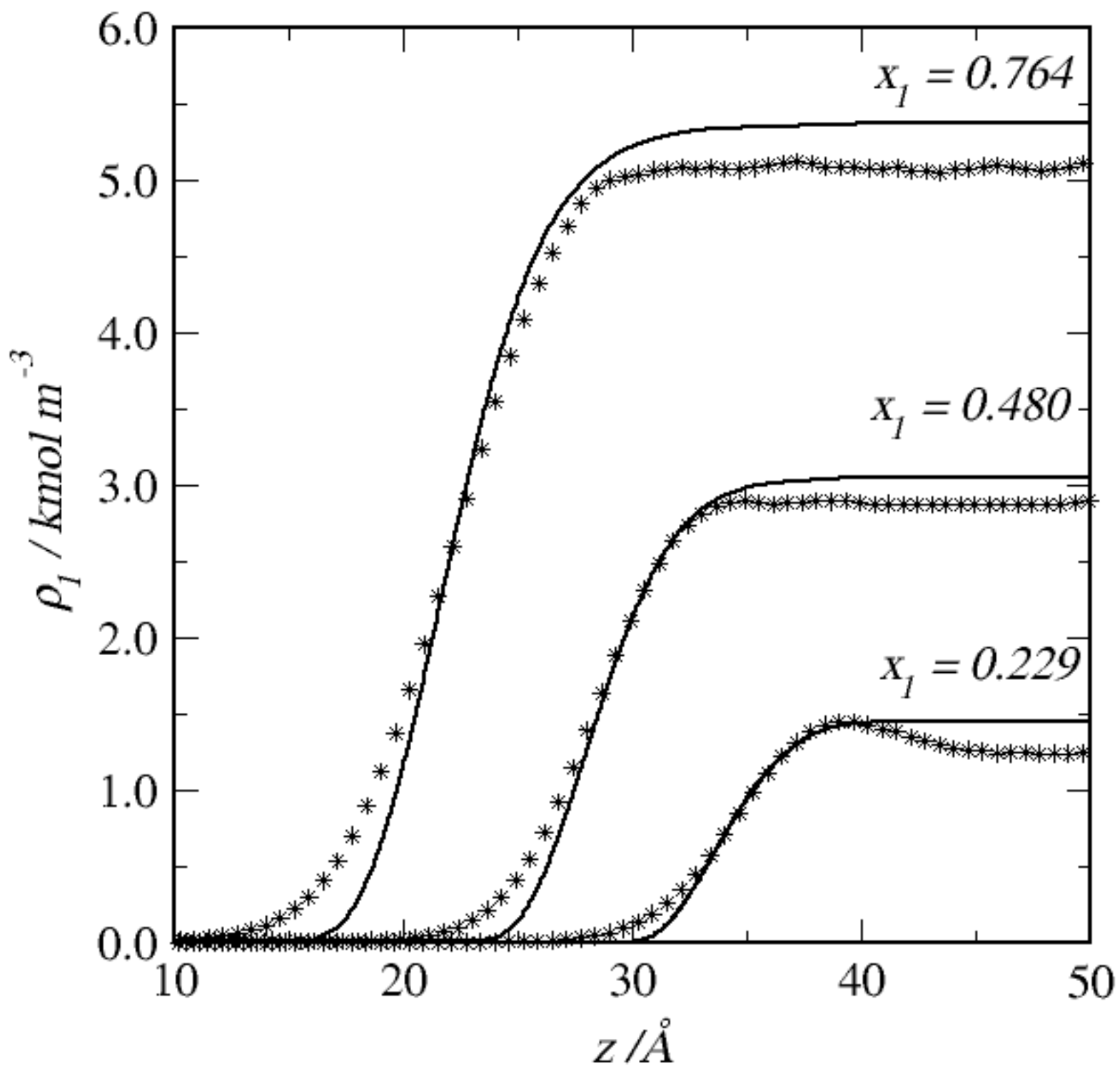

Figure 4.a 


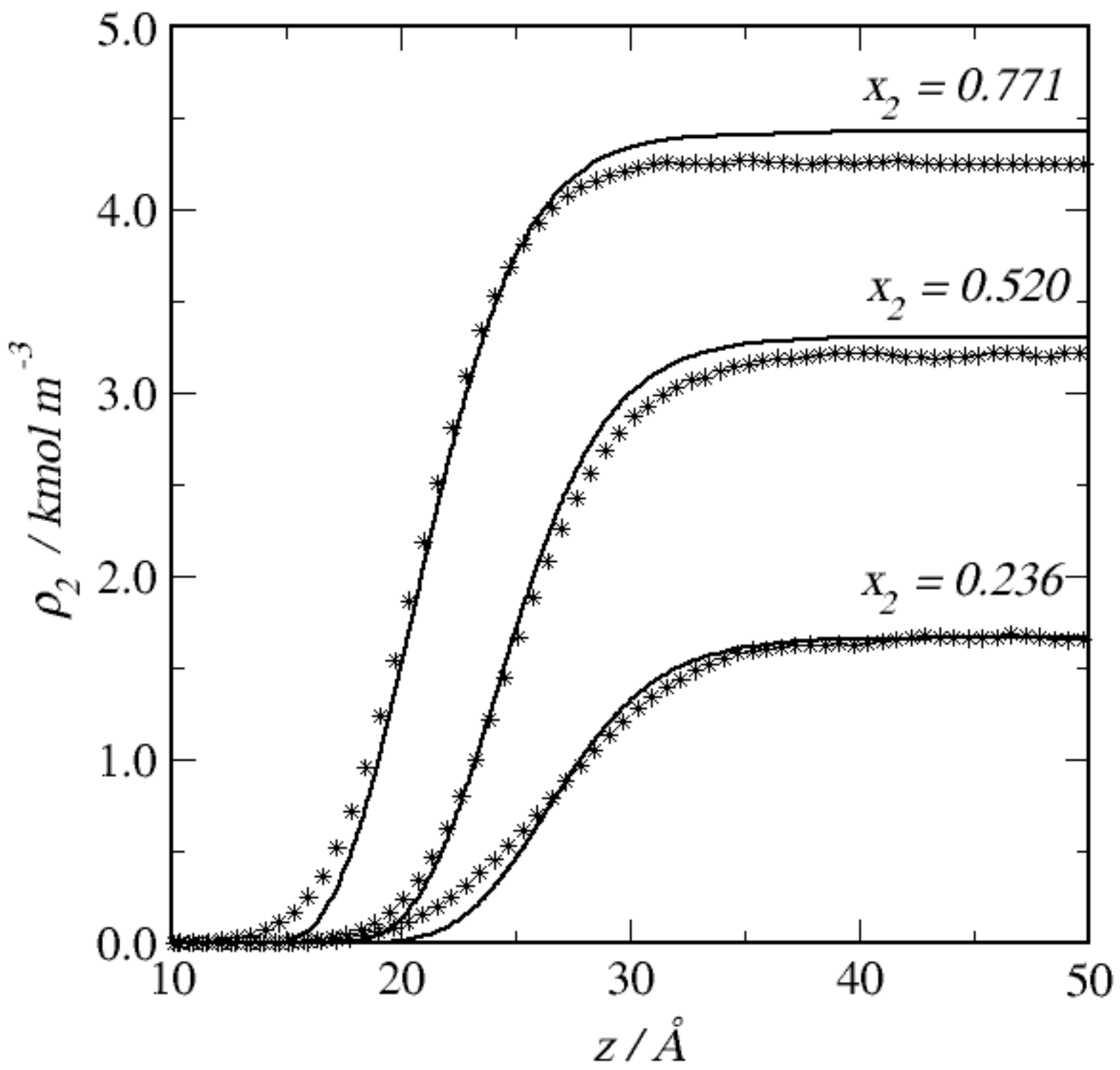

Figure 4.b 


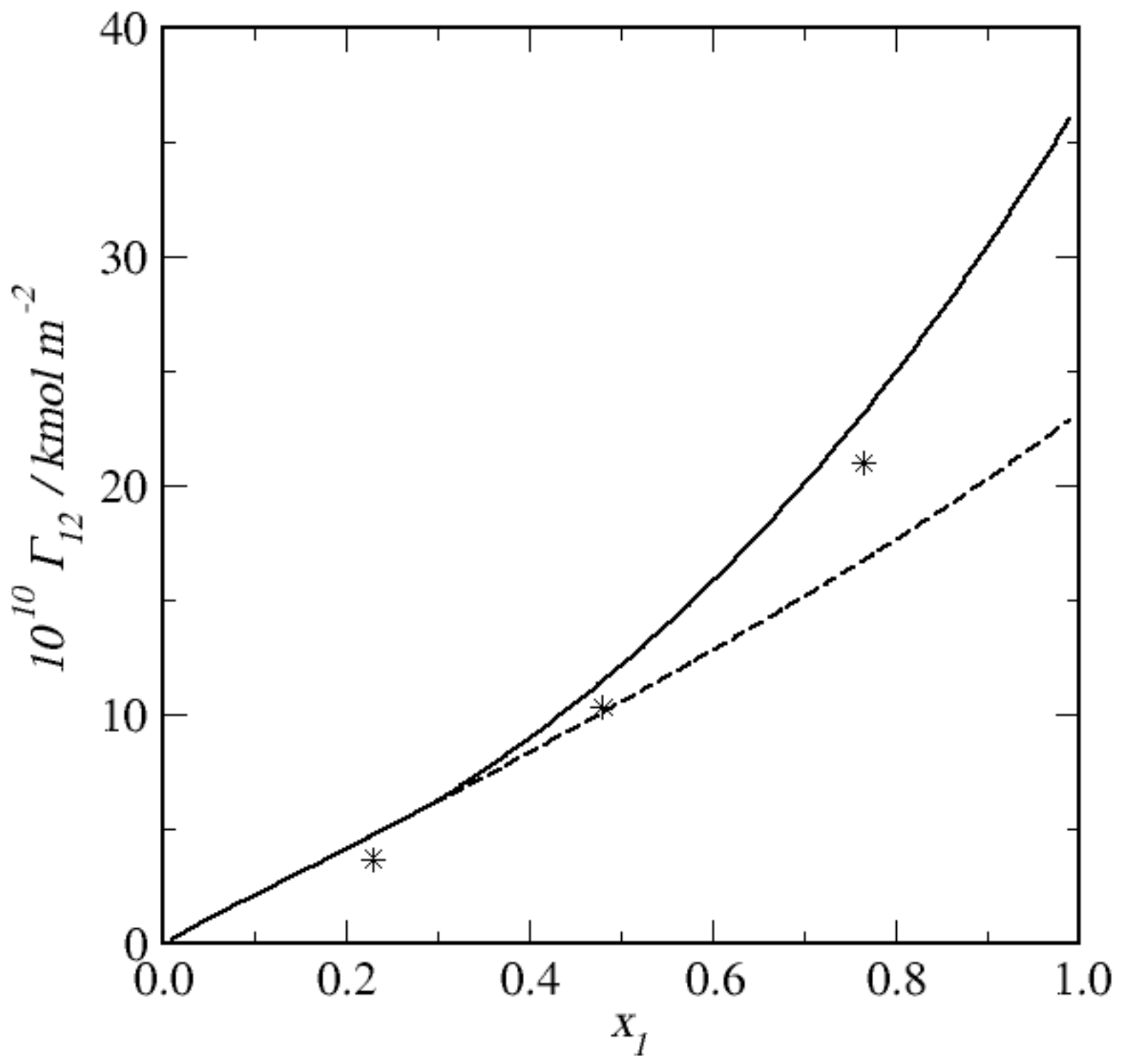

Figure 5 


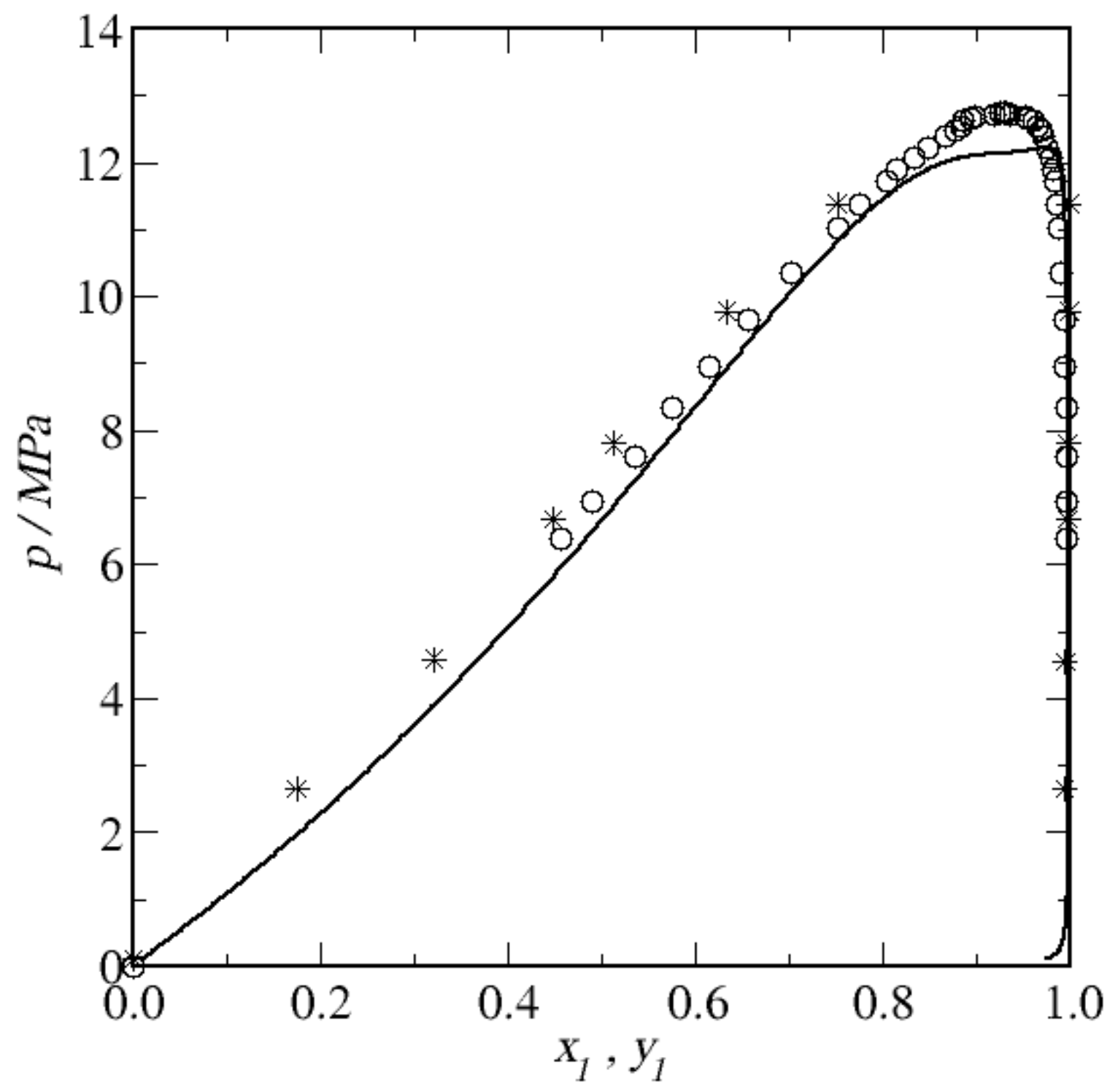

Figure 6 


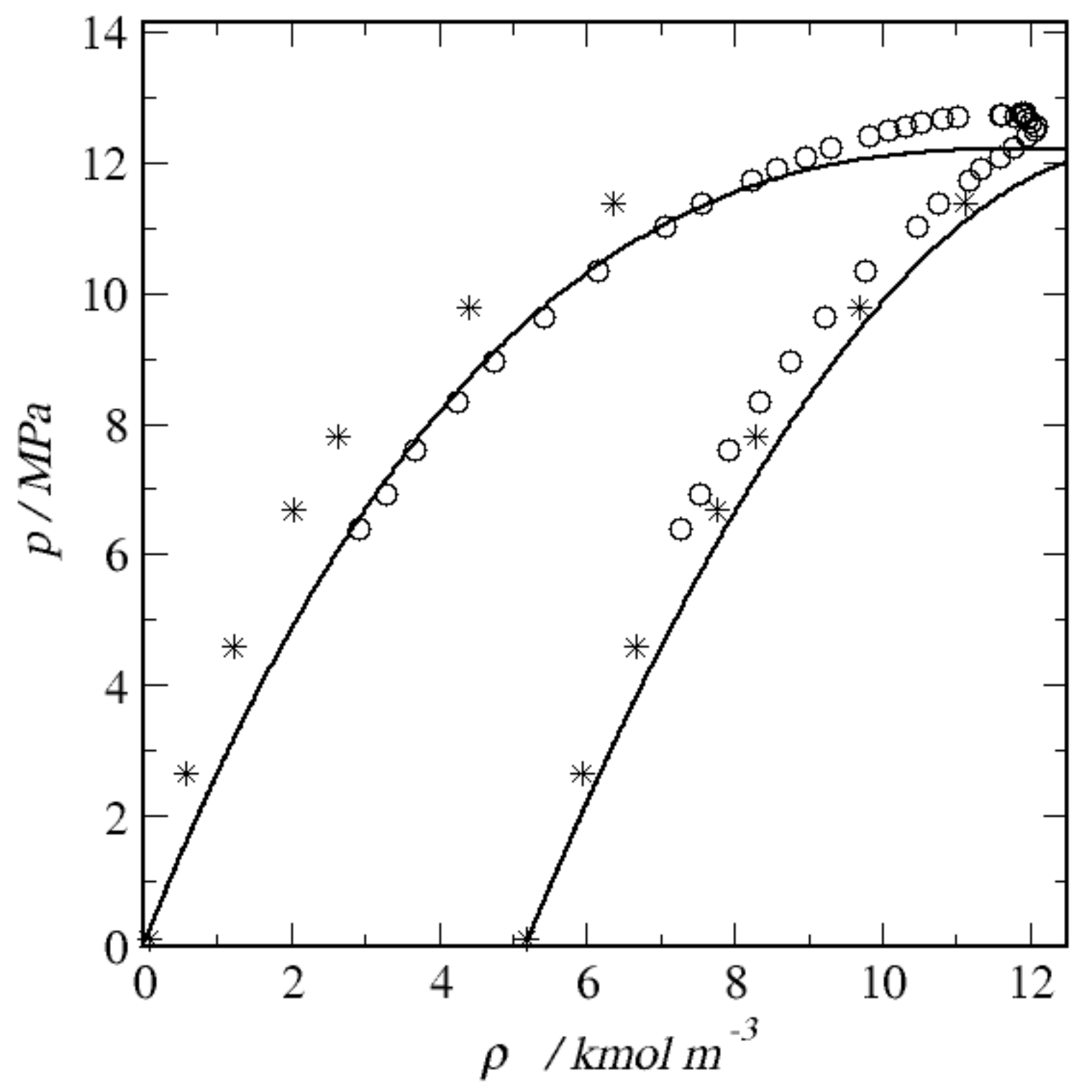

Figure 7 


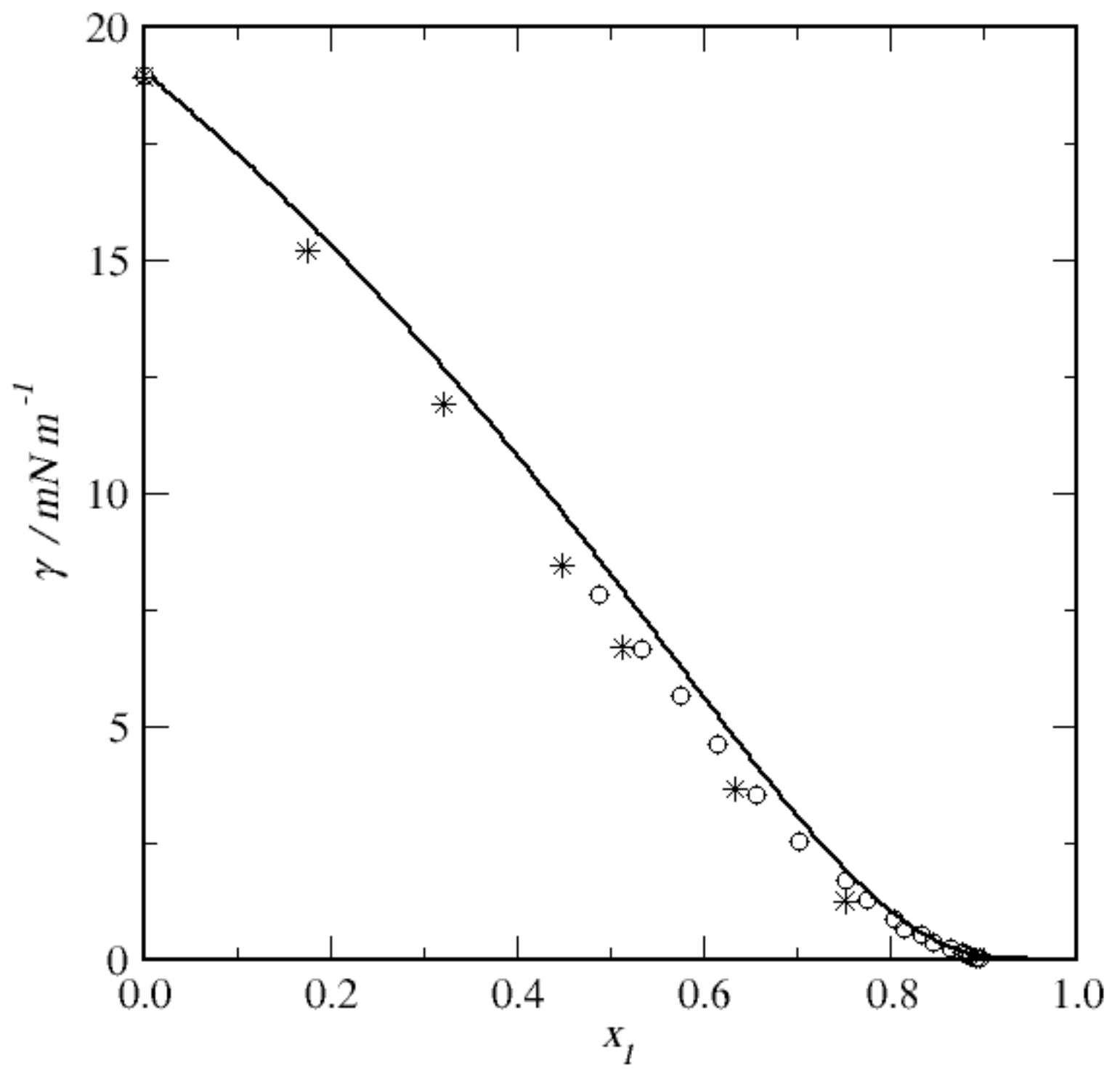

Figure 8 


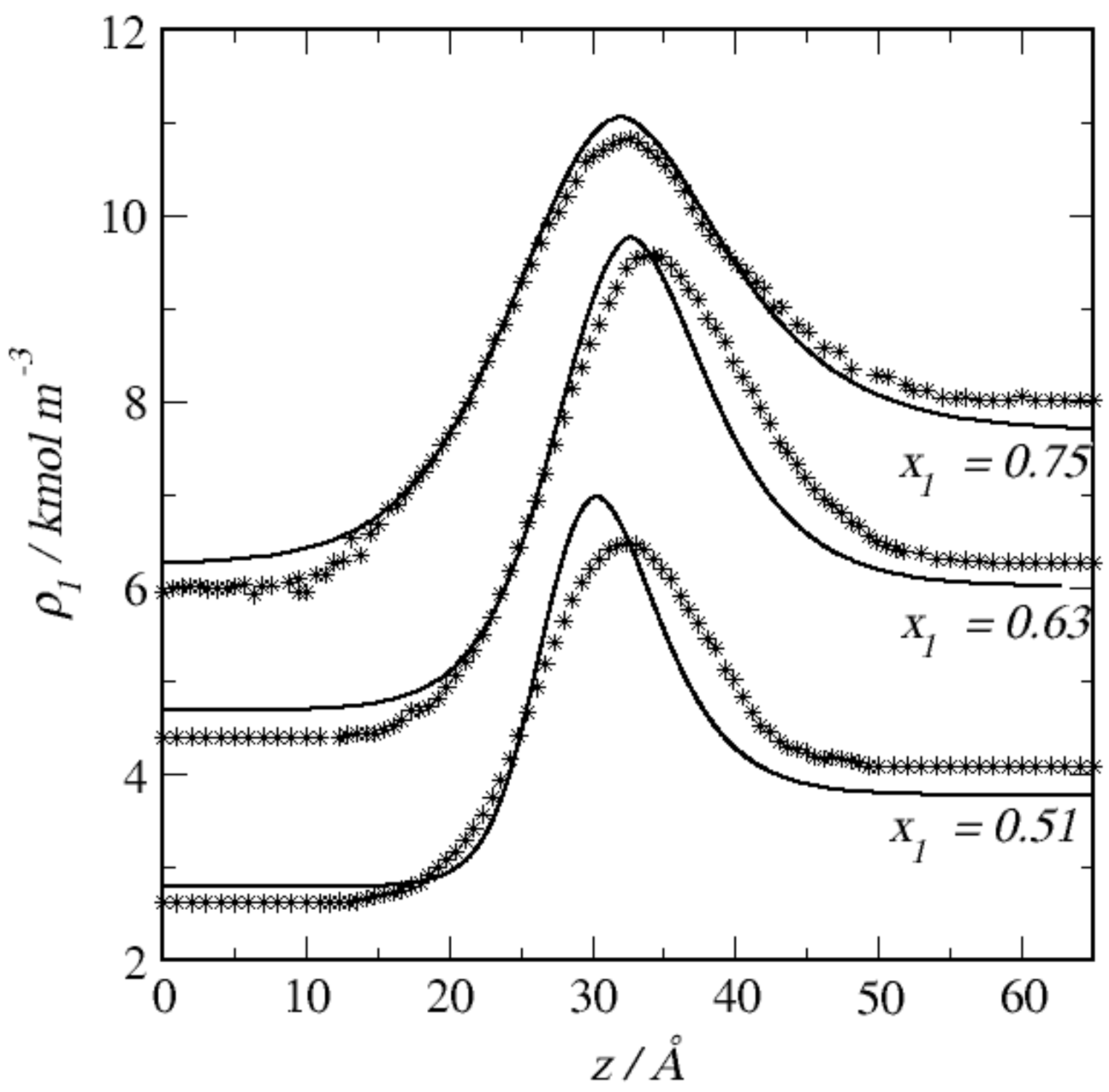

Figure 9.a 


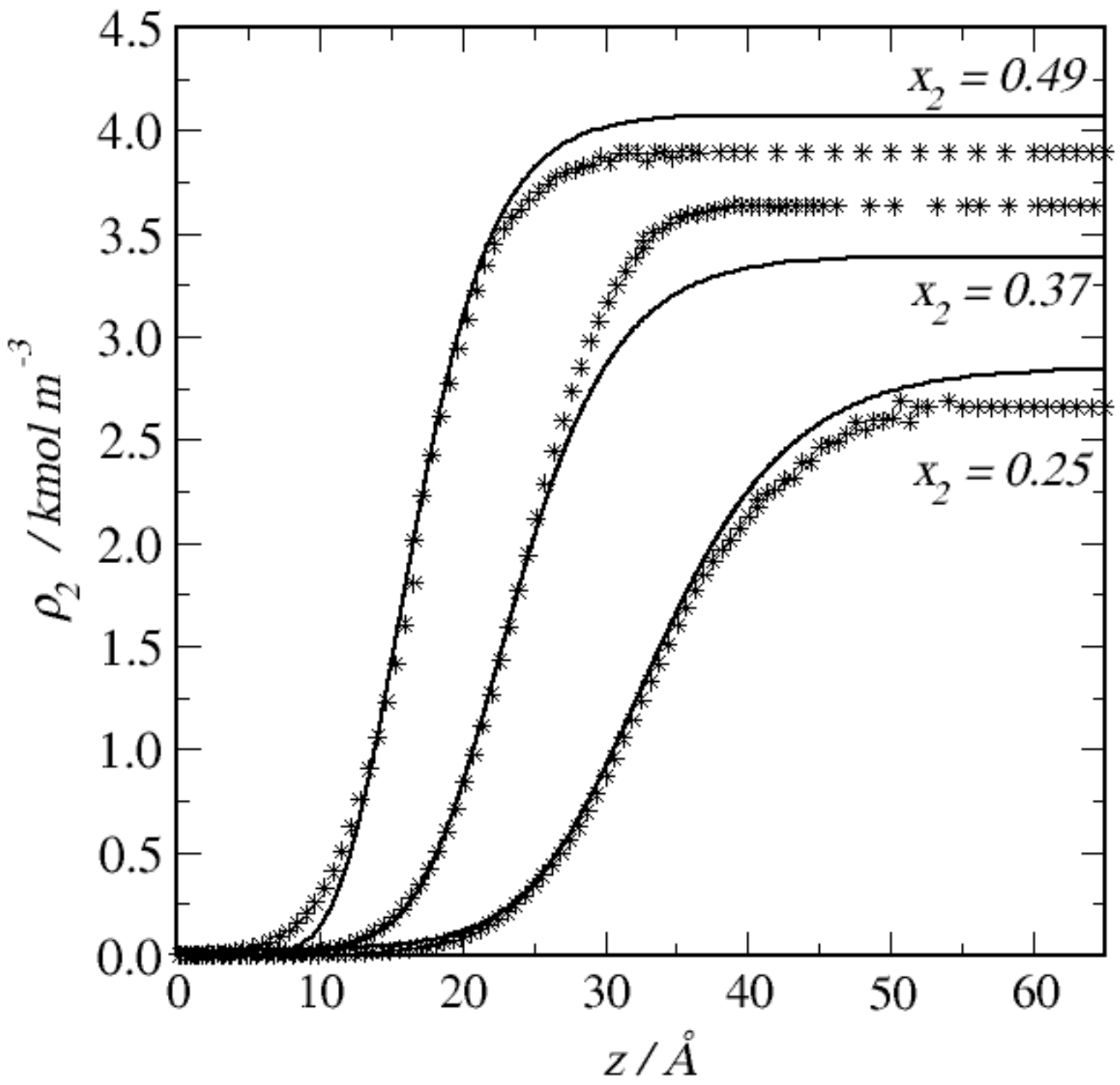

Figure 9.b 


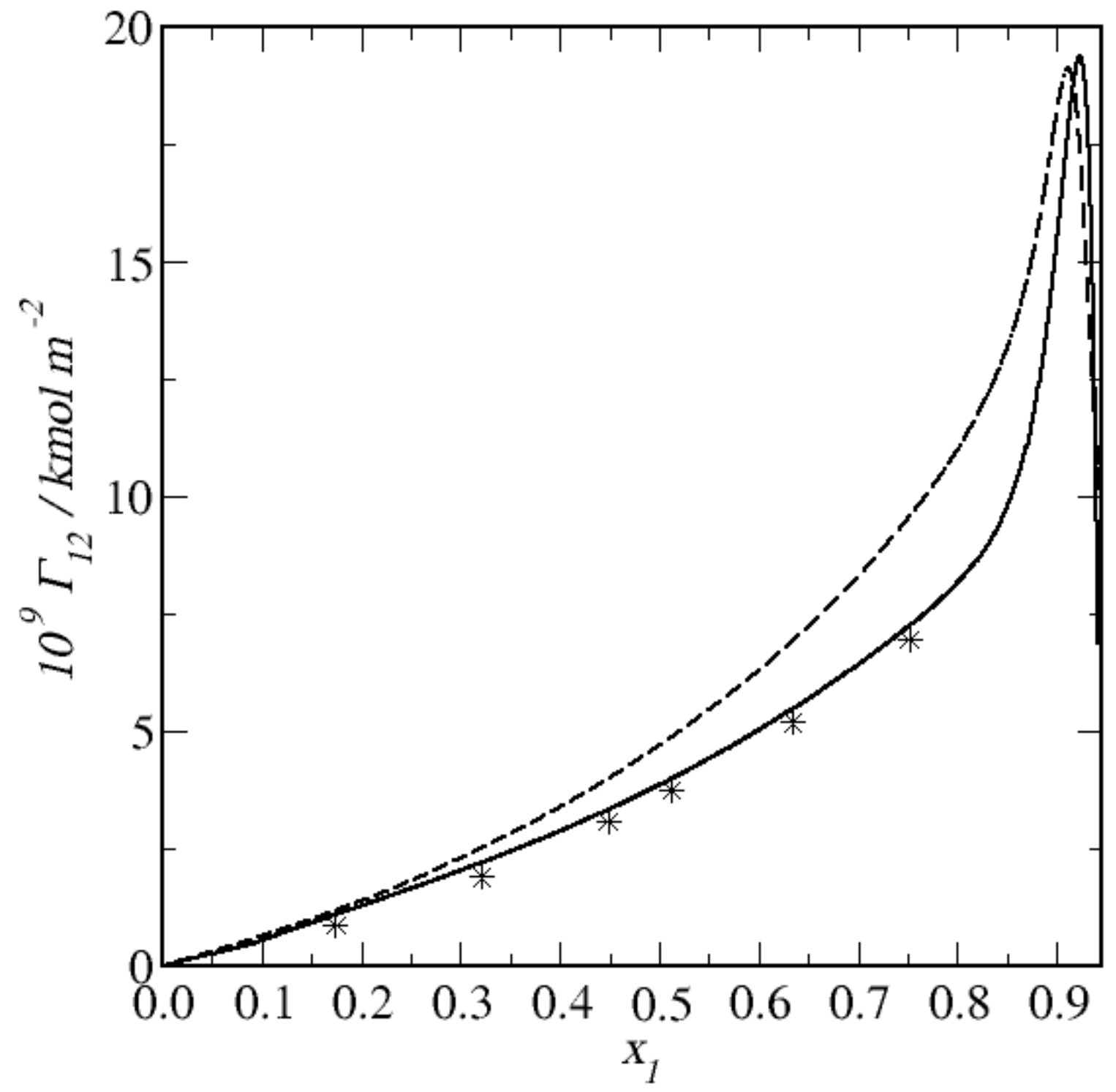

Figure 10 


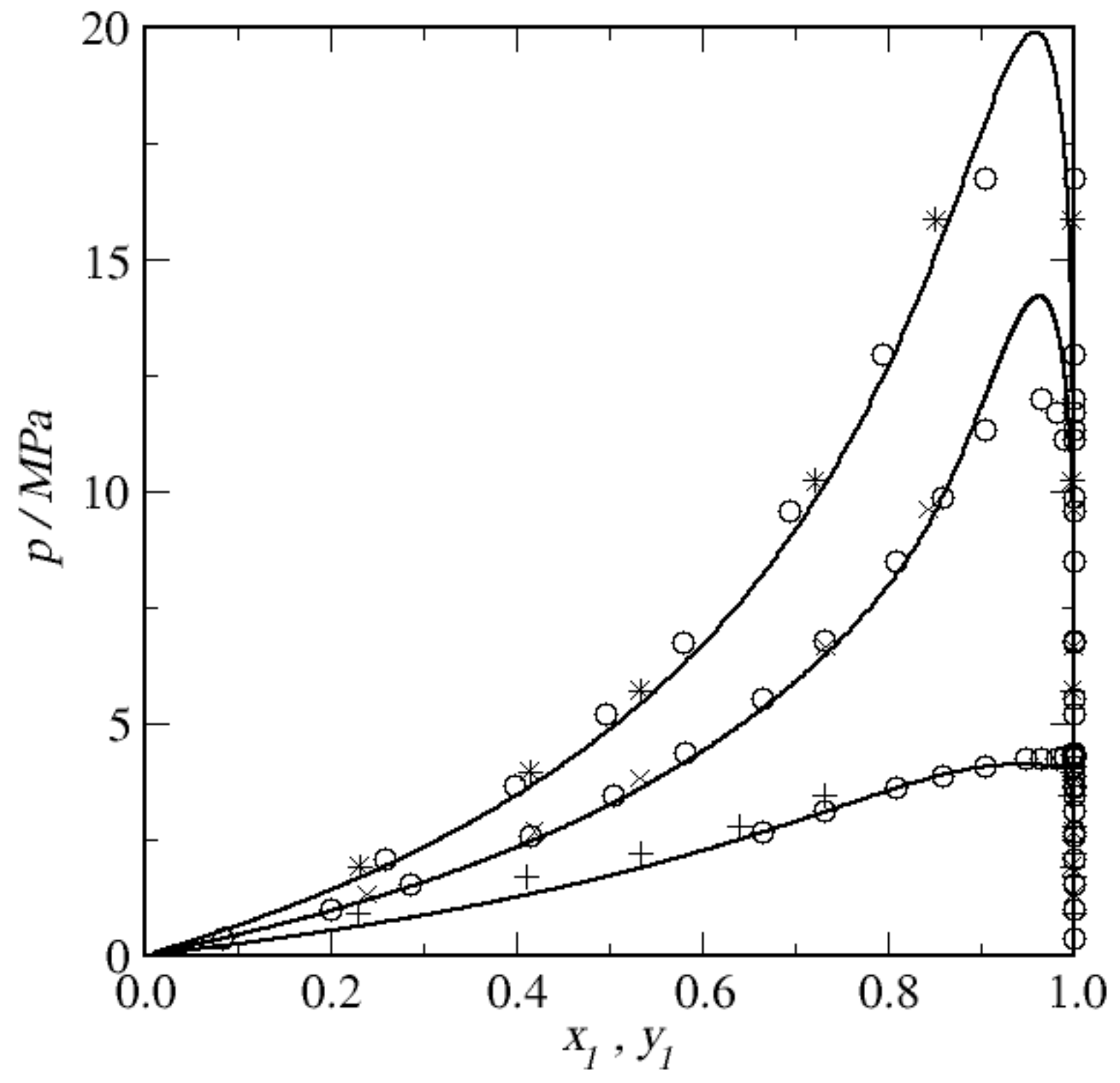

Figure 11 


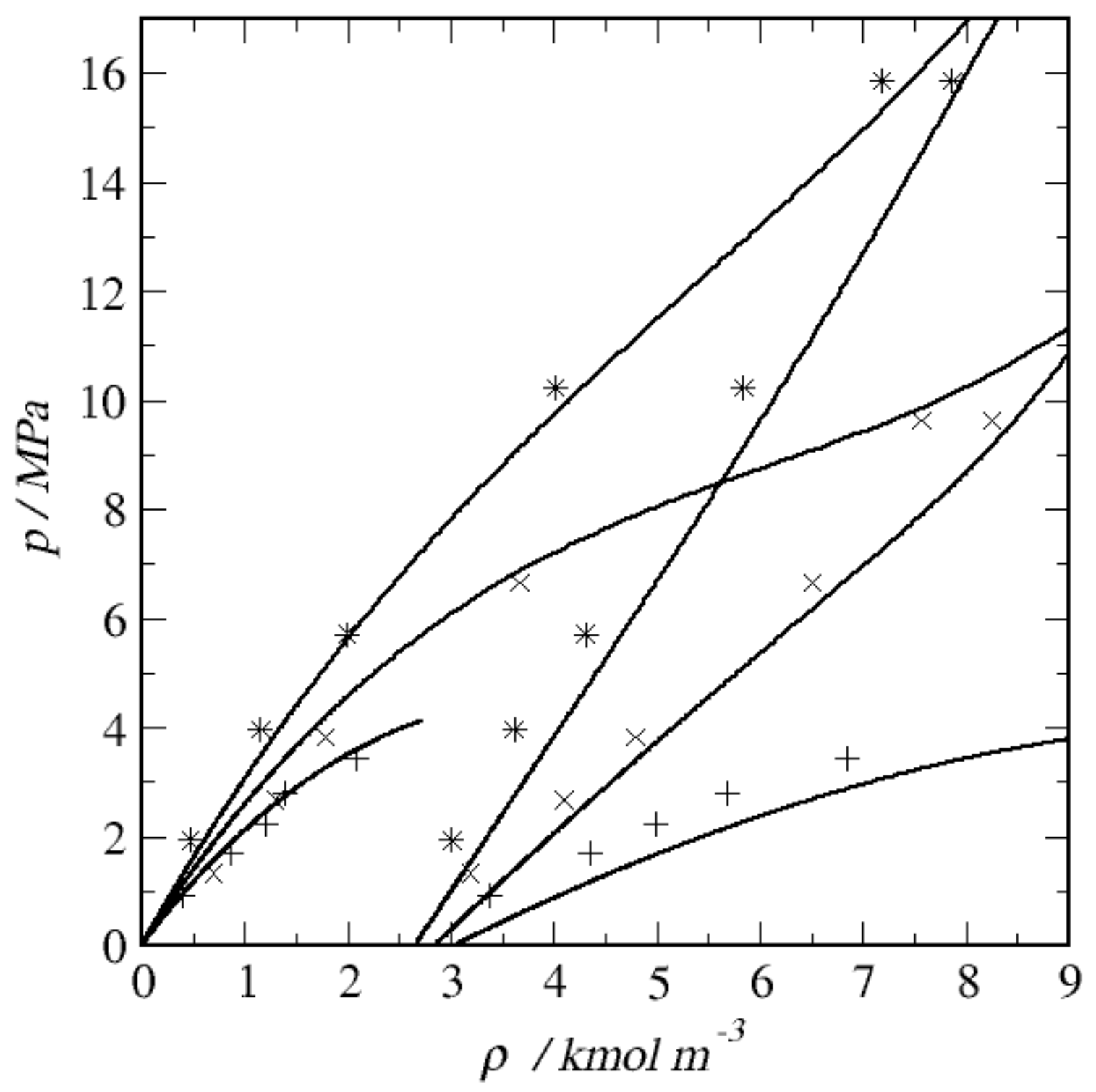

Figure 12 


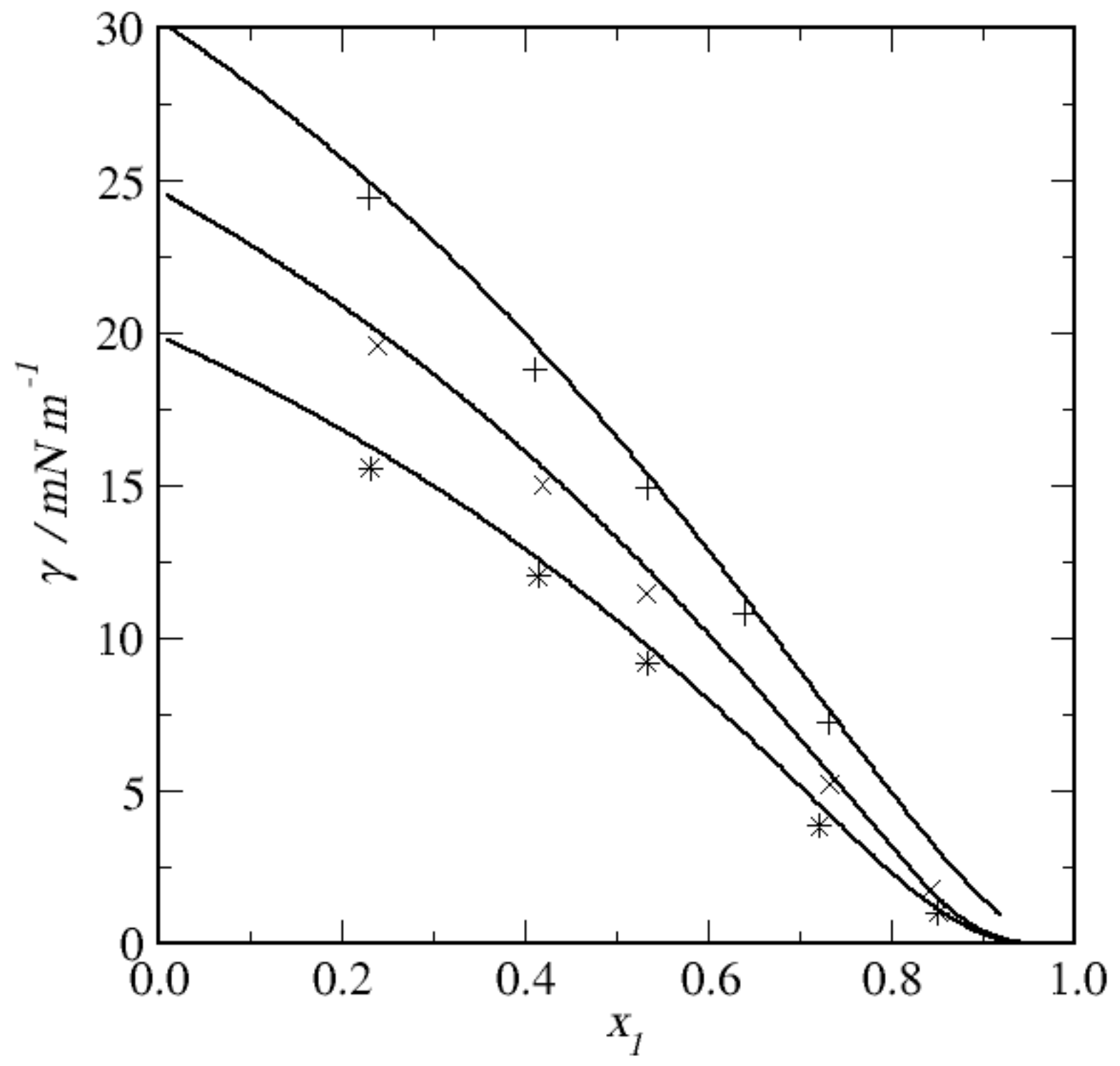

Figure 13 


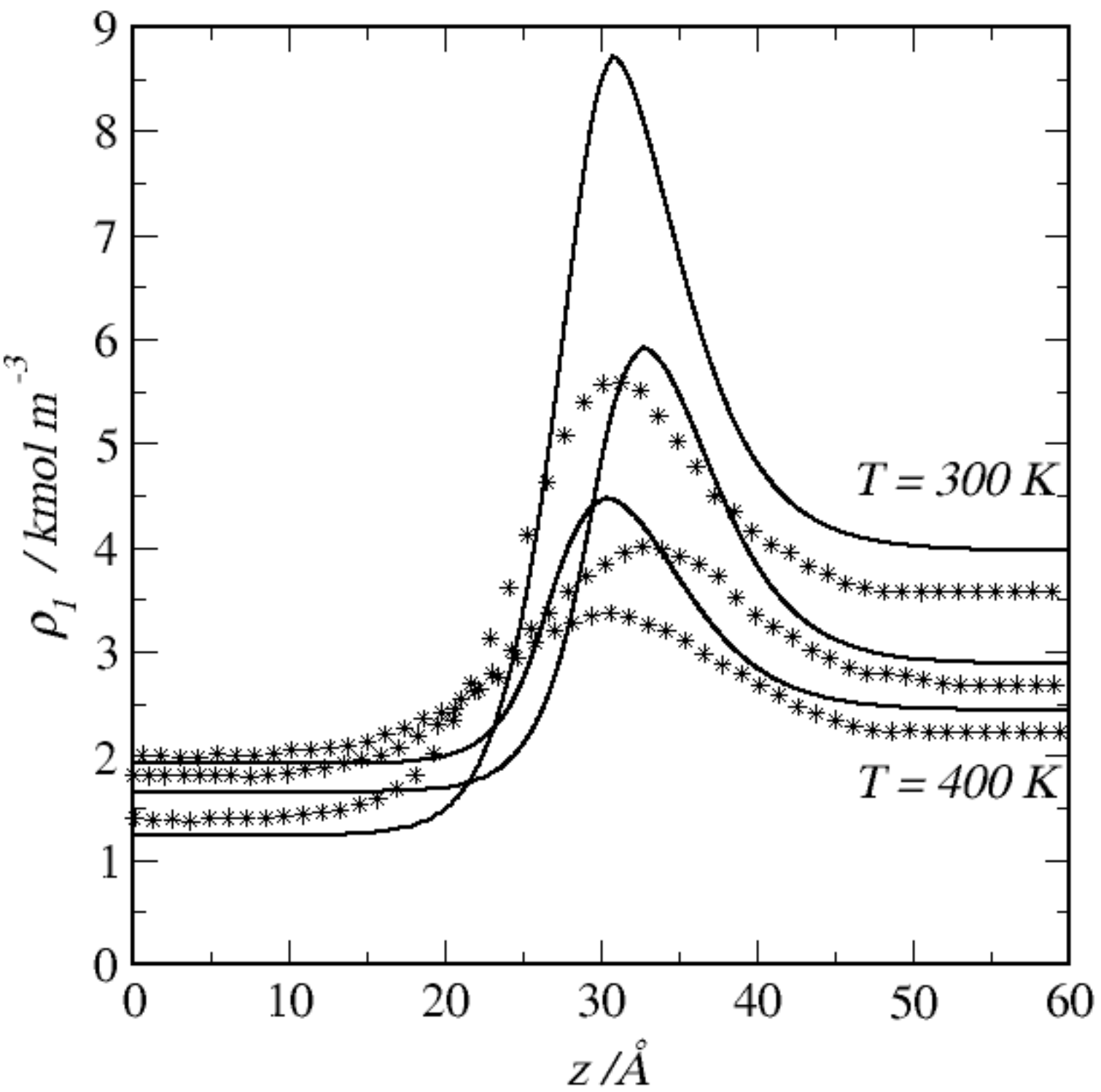

Figure 14.a 


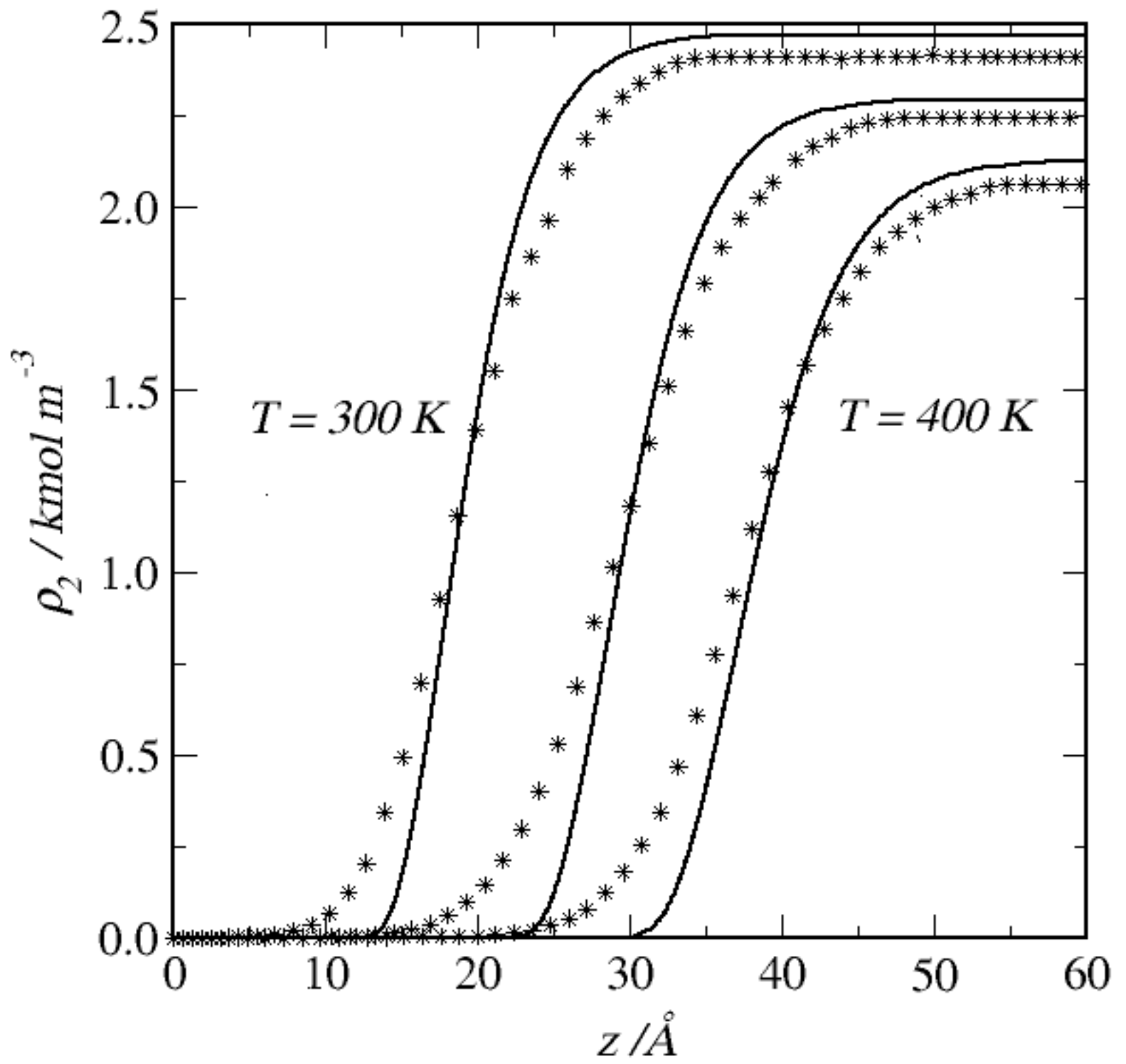

Figure 14.b 


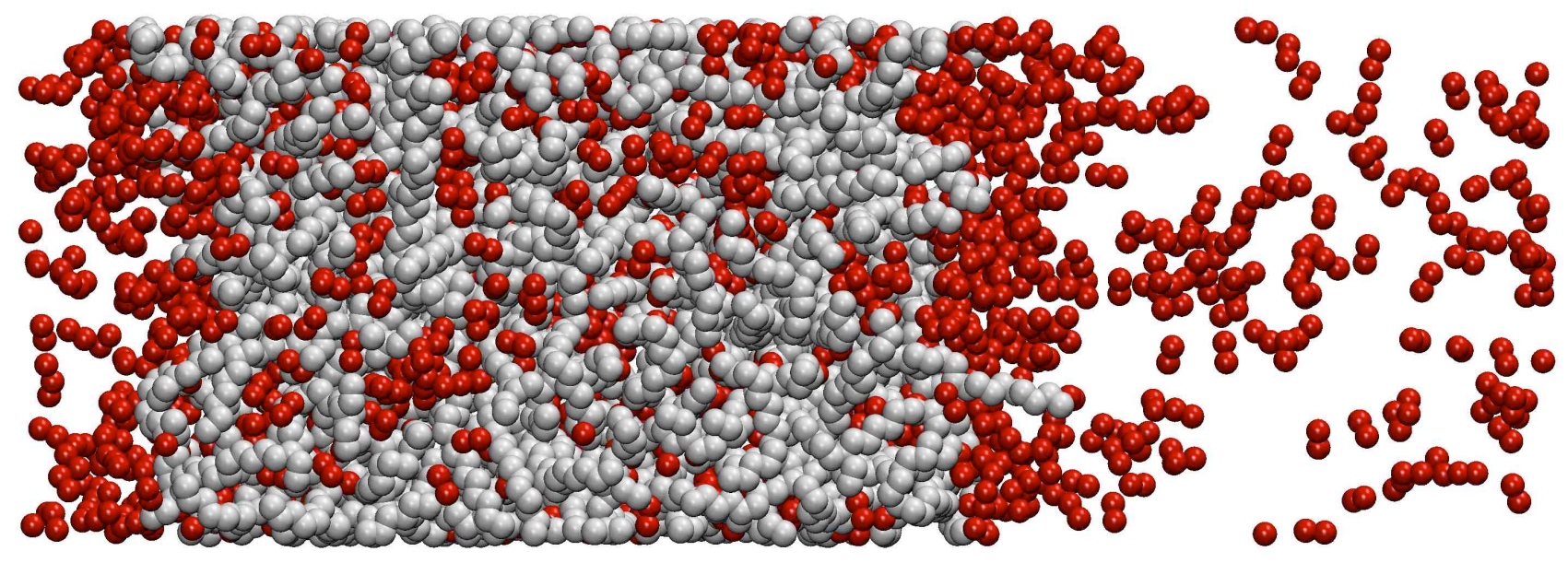

Figure 15 


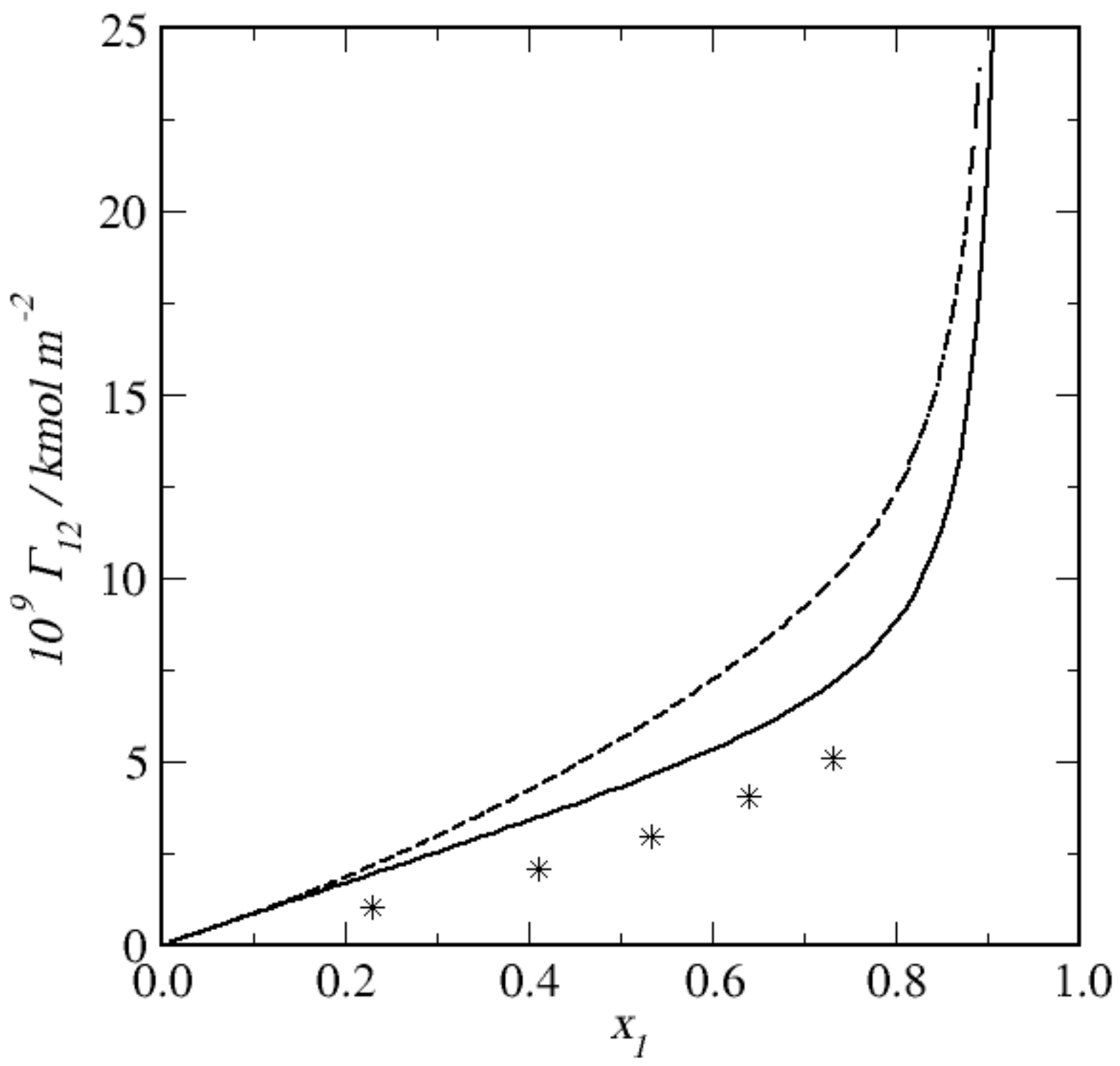

Figure 16. a 


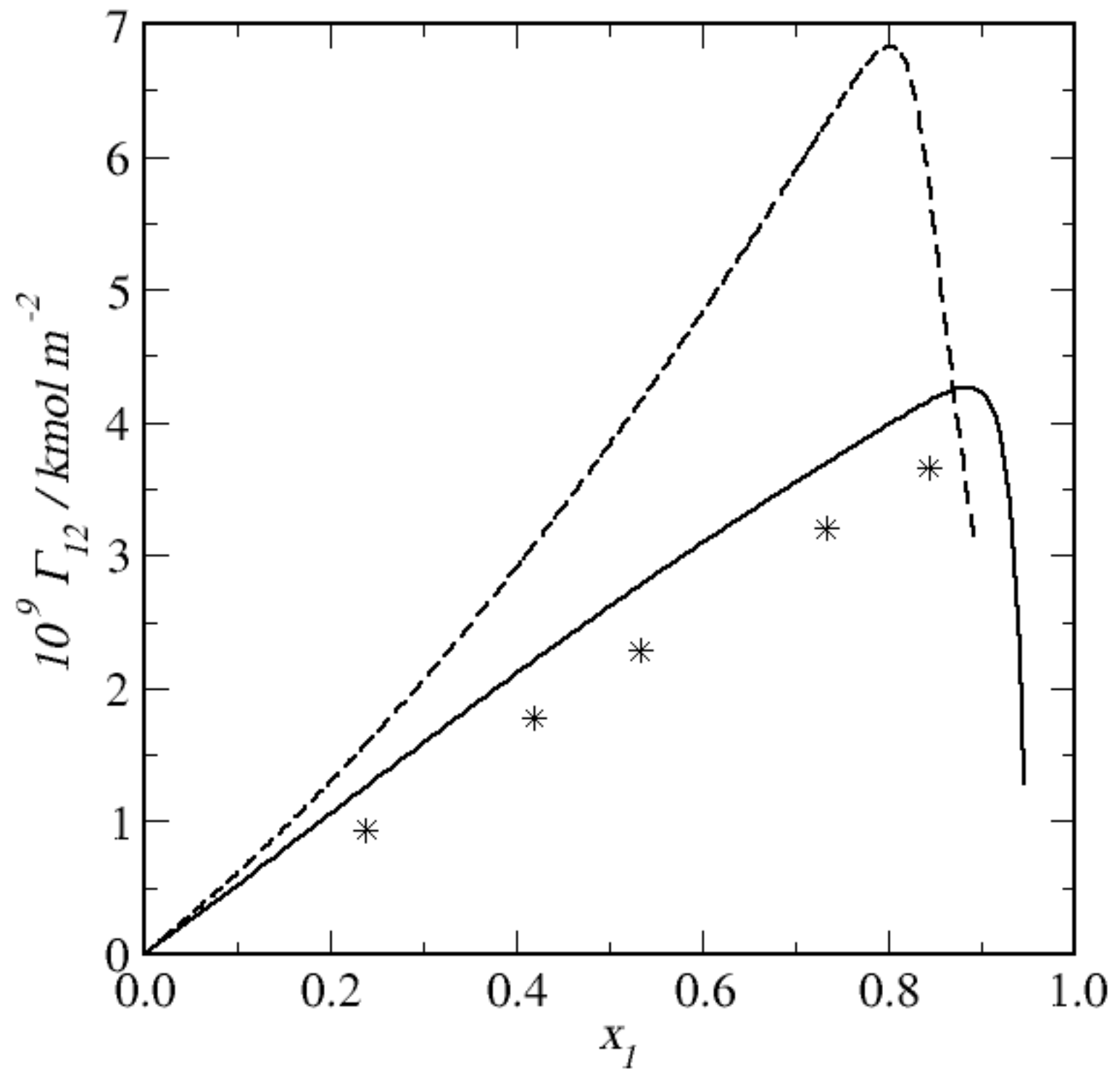

Figure 16. b 


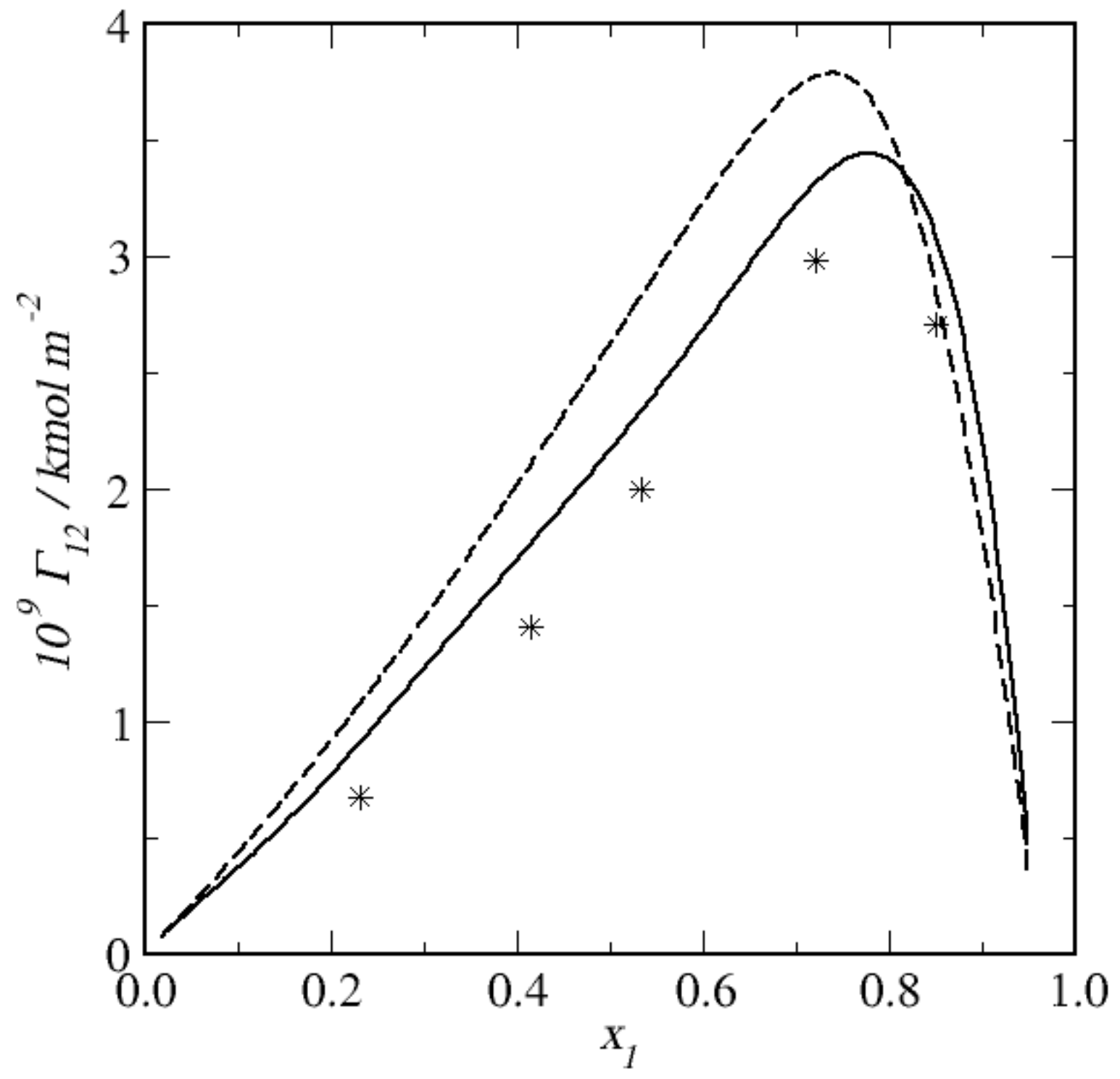

Figure 16. c 


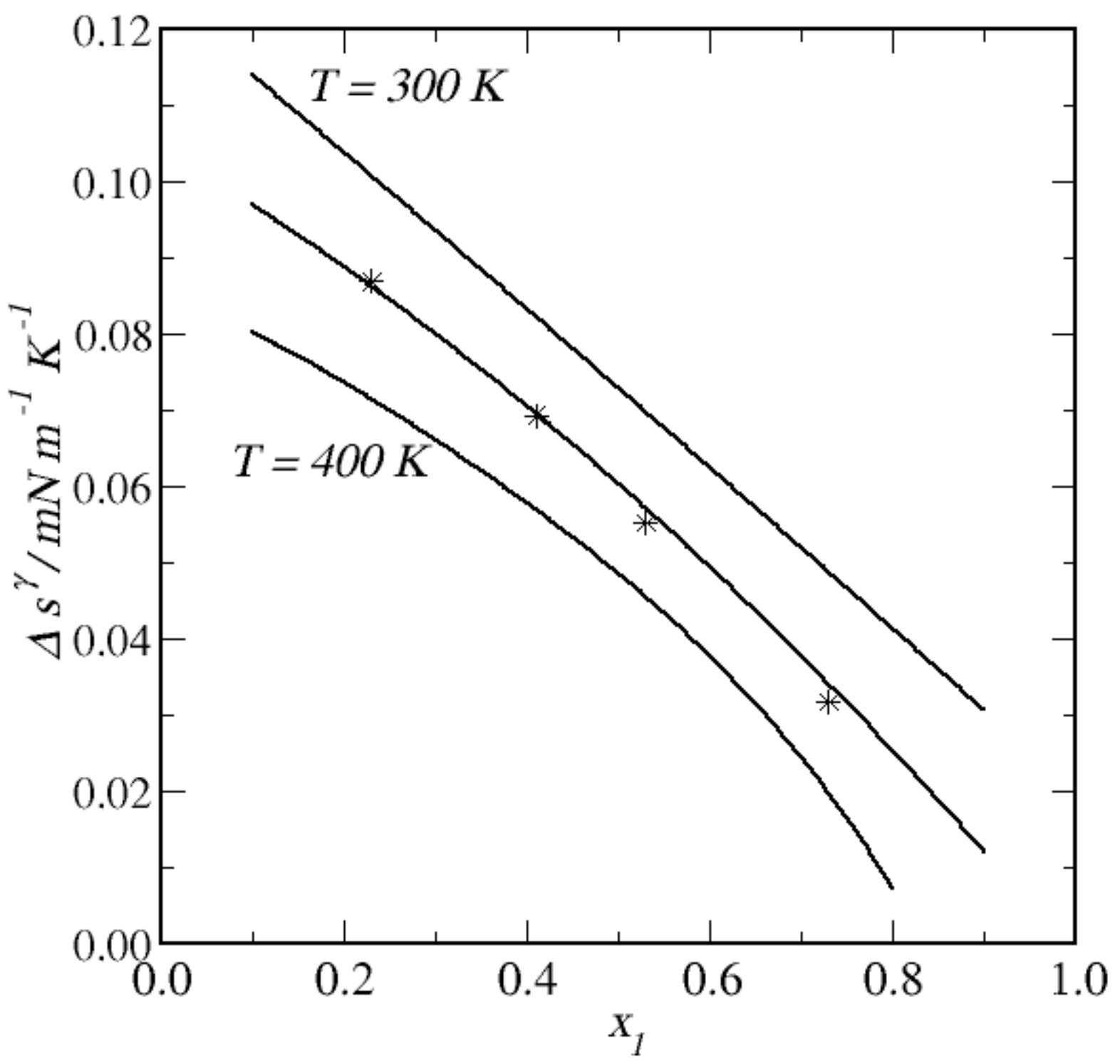

Figure 17 


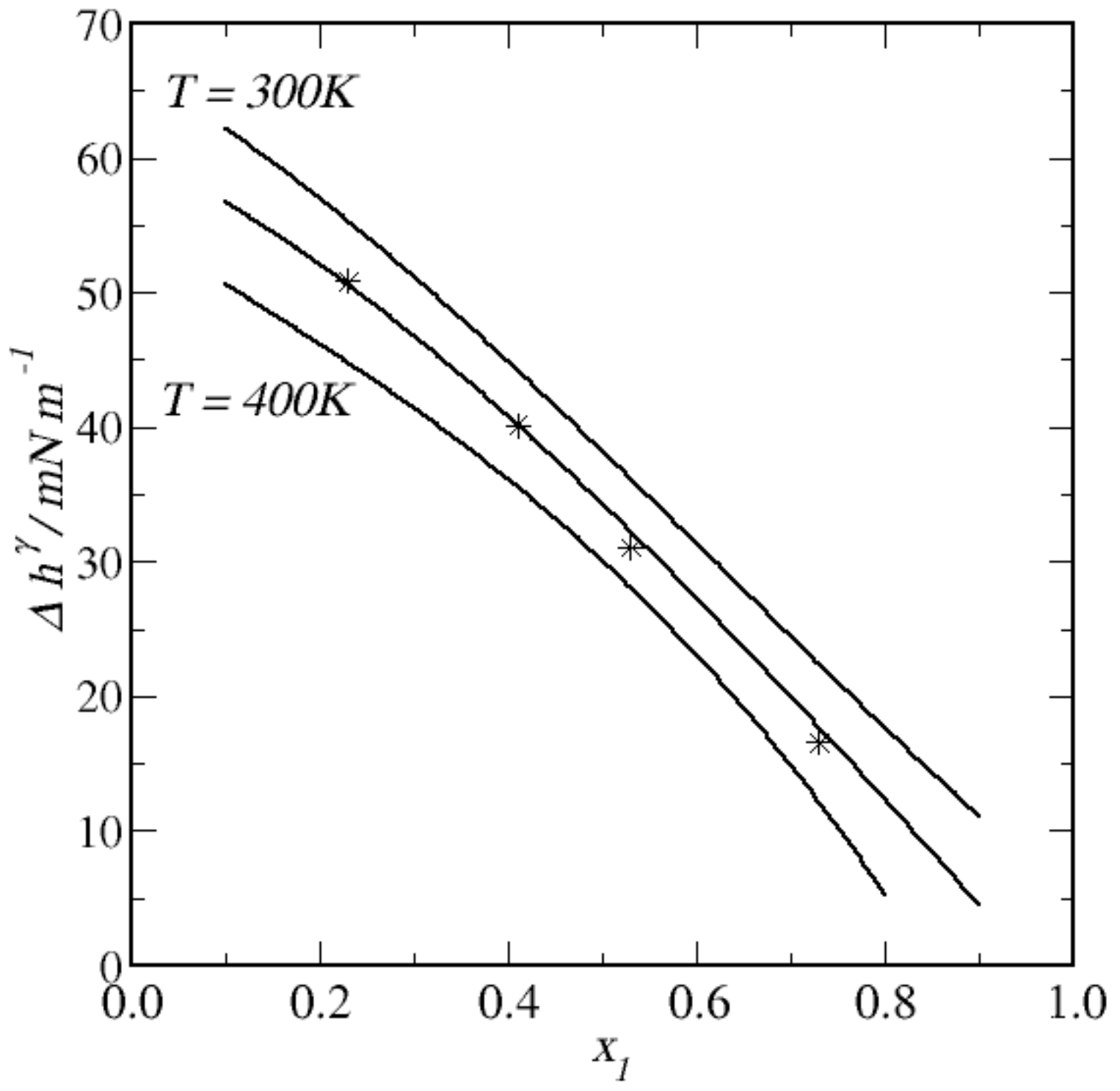

Figure 18 